\title{
Cluster Abell 520: a perspective based on member galaxies
}

\section{A cluster forming at the crossing of three filaments?}

\author{
M. Girardi ${ }^{1,2}$, R. Barrena ${ }^{3}$, W. Boschin ${ }^{1,4}$, and E. Ellingson ${ }^{5}$ \\ 1 Dipartimento di Astronomia of the Università degli Studi di Trieste, via Tiepolo 11, 34143 Trieste, Italy \\ e-mail: girardi@oats.inaf.it \\ 2 INAF - Osservatorio Astronomico di Trieste, via Tiepolo 11, 34143 Trieste, Italy \\ 3 Instituto de Astrofísica de Canarias, C/vía Láctea s/n, 38205 La Laguna (Tenerife), Canary Islands, Spain \\ ${ }^{4}$ Fundación Galileo Galilei - INAF, Rambla José Ana Fernández Perez 7, 38712 Breña Baja (La Palma), Canary Islands, Spain \\ 5 Center for Astrophysics and Space Astronomy, 389 UCB, University of Colorado, Boulder, CO 80309, USA
}

Received 9 July 2008 / Accepted 10 September 2008

\section{ABSTRACT}

\begin{abstract}
Context. The connection of cluster mergers with the presence of extended, diffuse radio sources in galaxy clusters is still debated. An interesting case is the rich, merging cluster Abell 520, containing a radio halo. A recent gravitational analysis has shown in this cluster the presence of a massive dark core suggested to be a possible problem for the current cold dark matter paradigm.

Aims. We aim to obtain new insights into the internal dynamics of Abell 520 analyzing velocities and positions of member galaxies. Methods. Our analysis is based on redshift data for 293 galaxies in the cluster field obtained combining new redshift data for 8 galaxies acquired at the TNG with data obtained by CNOC team and other few data from the literature. We also use new photometric data obtained at the INT telescope. We combine galaxy velocities and positions to select 167 cluster members around $z \sim 0.201$. We analyze the cluster structure using the weighted gap analysis, the KMM method, the Dressler-Shectman statistics and the analysis of the velocity dispersion profiles. We compare our results with those from X-ray, radio and gravitational lensing analyses.

Results. We compute a global line-of-sight (LOS) velocity dispersion of galaxies, $\sigma_{v}=1066_{-61}^{+67} \mathrm{~km} \mathrm{~s}^{-1}$. We detect the presence of a high velocity group (HVG) with a rest-frame relative LOS velocity of $v_{\mathrm{rf}} \sim 2000 \mathrm{~km} \mathrm{~s}^{-1}$ with respect to the main system (MS). Using two alternative cluster models we estimate a mass range $M\left(<1 h_{70}^{-1} \mathrm{Mpc}\right)=(4.0-9.6) \times 10^{14} h_{70}^{-1} M_{\odot}$. We also find that the MS shows evidence of subclumps along two preferred directions. The main, complex structure $\mathcal{N} \mathcal{E} 1+\mathcal{N} \mathcal{E} 2$ (with a velocity comparable to that of the MS) and the $\mathcal{S} W$ structure (at $v_{\mathrm{rf}} \sim+1100 \mathrm{~km} \mathrm{~s}^{-1}$ ) define the NE-SW direction, the same of the merger suggested by $\mathrm{X}$-ray and radio data. The $\mathcal{E}$ and $\mathcal{W}$ structures (at $v_{\mathrm{rf}} \sim-1150$ and $v_{\mathrm{rf}} \sim-300 \mathrm{~km} \mathrm{~s}^{-1}$ ) define the E-W direction. Moreover, we find no dynamical trace of an important structure around the lensing dark core. Rather, the HVG and a minor MS group, having different velocities, are roughly centered in the same position of the lensing dark core, i.e. are somewhat aligned with the LOS.

Conclusions. We find that Abell 520 is definitely a very complex system. Our results suggest that we are looking at a cluster forming at the crossing of three filaments of the large scale structure. The filament aligned with the LOS and projected onto the center of the forming cluster might explain the apparent massive dark core shown by gravitational lensing analysis.
\end{abstract}

Key words. galaxies: clusters: individual: Abell 520 - galaxies: clusters: general - galaxies: distances and redshifts

\section{Introduction}

Clusters of galaxies are by now recognized to be not simple relaxed structures, but rather they are evolving via merging processes in a hierarchical fashion from poor groups to rich clusters. Much progress has been made in recent years in the observations of the signatures of merging processes (see Feretti et al. 2002, for a general review). A recent aspect of these investigations is the possible connection of cluster mergers with the presence of extended, diffuse radio sources: halos and relics. Cluster mergers have been suggested to provide the large amount of energy necessary for electron reacceleration and magnetic field amplification (Feretti 1999; Feretti 2002; Sarazin 2002). However, the question is still debated since the diffuse radio sources are quite uncommon and only recently we can study these phenomena on the basis of a sufficient statistics (few dozen clusters up to $z \sim 0.3$, e.g. Giovannini et al. 1999; see also Giovannini \& Feretti 2002; Feretti 2005).
Growing evidence of the connection between diffuse radio emission and cluster merging is based on X-ray data (e.g. Böhringer \& Schuecker 2002; Buote 2002). Studies based on a large number of clusters have found a significant relation between the radio and the X-ray surface brightness (Govoni et al. 2001a,b) and connections between the presence of radiohalos/relics and irregular and bimodal X-ray surface brightness distribution (Schuecker et al. 2001).

Optical data are a powerful way to investigate the presence and the dynamics of cluster mergers (e.g. Girardi \& Biviano 2002), too. The spatial and kinematical analysis of member galaxies allow us to detect and measure the amount of substructure, to identify and analyze possible pre-merging clumps or merger remnants. This optical information is really complementary to X-ray information since galaxies and intra-cluster medium react on different time scales during a merger (see, e.g. numerical simulations by Roettiger et al. 1997). In this context we are conducting an intensive observational and data analysis program to study the internal dynamics of radio clusters by 


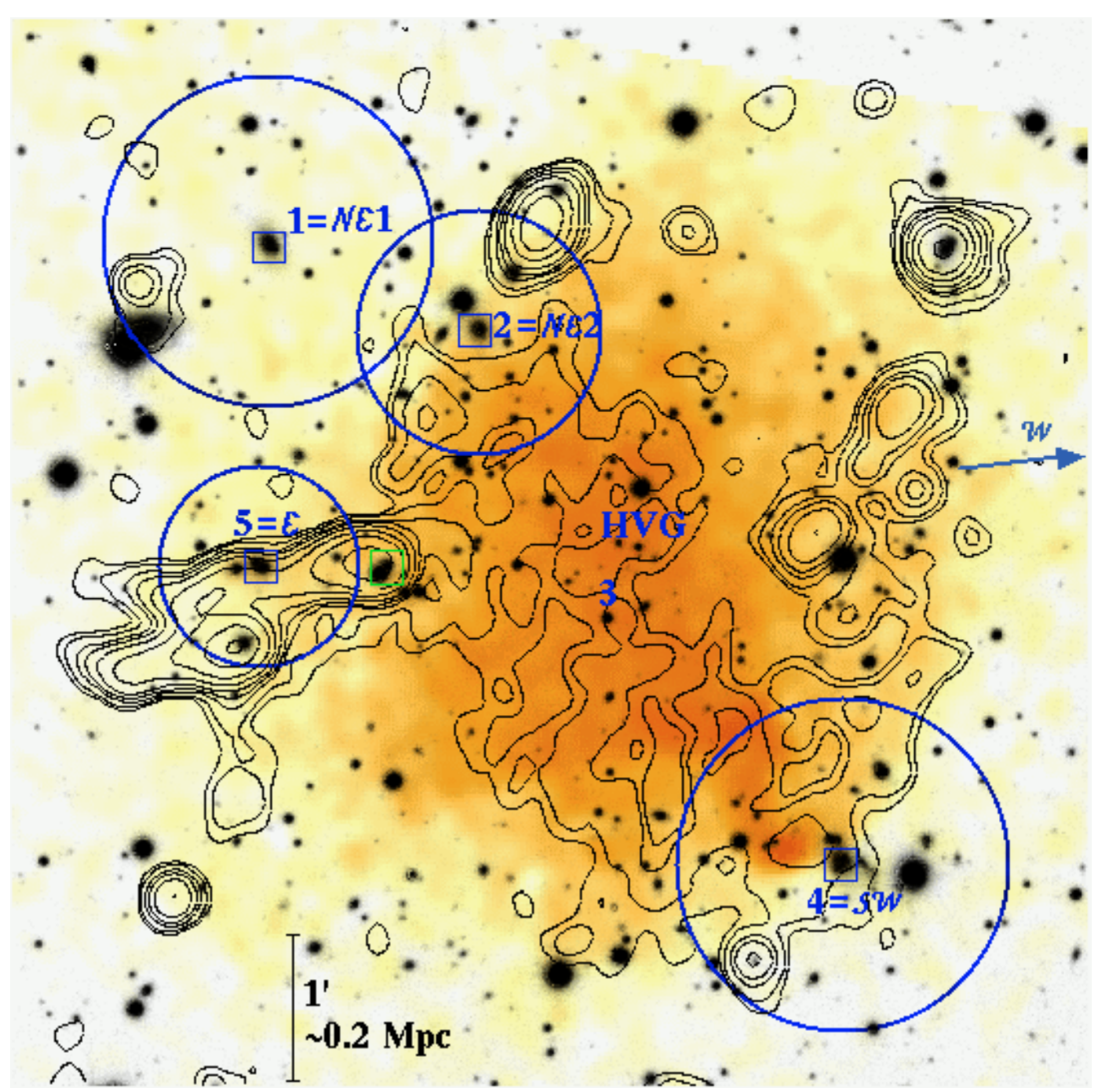

Fig. 1. Multiwavelength picture of A520 (North is at the top and East to the left). A smoothed Chandra 0.5-2 keV image (orange and yellow colors) of the central region of A520 (courtesy of Markevitch - Markevitch et al. 2005, X-ray point sources are removed) is superimposed to a $r^{\prime}$-band image taken with the WFC camera of the INT. The contour levels of a VLA radio image at $1.4 \mathrm{GHz}$ (courtesy of Govoni - Govoni et al. 2001b) are shown, too. Main structures recovered by our analysis are highlighted (see Sect. 5, for more details). Label HVG indicates the center of the high velocity group having a relative LOS velocity of $v_{\mathrm{rf}} \sim 2000 \mathrm{~km} \mathrm{~s}^{-1}$ with respect to the main system (MS). Dark blue circles and numbers highlight the positions of the five peaks in the lensing mass distribution found by M07. The size of the circles indicate the regions where we find evidence for an individual, dynamically important structure of the MS. The name of each structure is indicated by the label close to the corresponding M07 peak number and the dark blue small square indicates the central, luminous galaxy (i.e. galaxies IDs 204, 170, 106 and 205 for $\mathcal{N} \mathcal{E} 1, \mathcal{N} \mathcal{E} 2, \mathcal{S} W$ and $\mathcal{E}$, respectively). Finally, the green square indicates a head-tail radiogalaxy.

using member galaxies. Our program concerns both massive clusters, where diffuse radio emissions are more frequently found (e.g. Barrena et al. 2007b, and references therein), and low-mass galaxy systems (Boschin et al. 2008 ${ }^{1}$ ).

During our observational program we have conducted an intensive study of the massive cluster Abell 520 (hereafter A520). This cluster shows a radio halo, discovered by Giovannini et al. (1999), having a low surface brightness with a clumpy structure slightly elongated in the NE-SW direction (Govoni et al. 2001b, 2004, see Fig. 1).

A520, also known as MS $0451+02$ in the EMSS catalog (Gioia et al. 1990), is a fairly rich, X-ray luminous, and hot cluster, with a galaxy population characterized by a high velocity

${ }^{1}$ Please visit the web site of the DARC (Dynamical Analysis of Radio Clusters) project: http://adlibitum.oat.ts.astro.it/ girardi/darc dispersion: Abell richness class $=1$ (Abell et al. 1989), $L_{\mathrm{X}}(0.1-$ $2.4 \mathrm{keV})=14.20 \times 10^{44} h_{50}^{-2} \mathrm{erg} \mathrm{s}^{-1}$ (Ebeling et al. 1996); $T_{\mathrm{X}}=7.1 \pm 0.7 \mathrm{keV}$ (Chandra data, Govoni et al. 2004); $\sigma_{v}=$ $(988 \pm 76) \mathrm{km} \mathrm{s}^{-1}$ (Carlberg et al. 1996).

First hints about the young dynamical status of this cluster came from both X-ray and optical data (Le Fevre et al. 1994; Gioia \& Luppino 1994, and references therein). The complexity of its structure was confirmed by analyses of ROSAT and Chandra X-ray data (Govoni et al. 2001b, 2004). In particular, new unprecedent insights were recovered from deep Chandra observations by Markevitch et al. (2005). They revealed a prominent bow shock indicating a cluster merger where a SW irregular structure consists of dense, cool pieces of a cluster core that has been broken up by ram pressure as it flew in from the NE direction (see Fig. 1). The overall structure of the radio halo seems connected with the cluster merger and may even suggest two distinct components, a mushroom with a stem and a cap, where the 
main stem component goes across the cluster along the NE-SW direction and the cap ends at the bow shock (Govoni et al. 2001b; Markevitch et al. 2005).

The complex structure of A520 was also confirmed by gravitational lensing analysis of Dahle et al. (2002), Mahdavi et al. (2007, hereafter M07) and Okabe \& Umetsu (2008). Okabe \& Umetsu (2008, based on Subaru data) found a general good agreement between mass and galaxy luminosity distribution. However, the detailed study of M07 based on the same Subaru data and additional CFHT data pointed out a less clear situation. M07 found four very significant peaks in the lensing mass distribution. Among these, peaks Nos. 1, 2 and 4 correspond to peaks in the galaxy distribution and give usual values for the mass-tolight ratio. Peak No. 3 corresponds to the central X-ray emission peak, but is largely devoid of galaxies. This peak is characterized by a very high mass-to-light value; thus to be referred as a "massive dark core". A region characterized by a somewhat low mass-to-light ratio exists, too (less significant peak No. 5). This displacement between galaxy and mass (i.e. dark matter, for the most part) remains very puzzling. In fact, galaxies and cold dark matter (CDM), being both treated as collisionless components, are expected to have similar behavior during a cluster merger. If confirmed by better observations, this situation would be difficult to explain within the widely accepted CDM paradigm of cosmological structure formation (see M07, for further discussions).

As for the analysis of the internal dynamics based on member galaxies, Proust et al. (2000) found some evidence of substructure using a sample of 21 galaxies, while the large data sample constructed by the Canadian Network for Observational Cosmology (hereafter CNOC) team (Carlberg et al. 1996; Yee et al. 1996) is still not exploited apart from few individual galaxies in M07. Recently, we have carried out spectroscopic observations at the TNG telescope giving new redshift data for 86 galaxies in the field of A520, as well as photometric observations at the INT telescope. Our present analysis is based on these optical data as well as on the large data sample obtained by CNOC.

This paper is organized as follows. We present our new optical data in Sect. 2 and the complete redshift catalog with the addition of CNOC and a few other data in Sect. 3. We present our results about global properties and substructure in Sect. 4. We furtherly analyze and discuss the dynamical status of A520 in Sect. 5. We draw our conclusions in Sect. 6.

Unless otherwise stated, we give errors at the $68 \%$ confidence level (hereafter c.l.). Throughout this paper, we use $H_{0}=70 h_{70} \mathrm{~km} \mathrm{~s}^{-1} \mathrm{Mpc}^{-1}$ in a flat cosmology with $\Omega_{0}=0.3$ and $\Omega_{\Lambda}=0.7$. In the adopted cosmology, $1^{\prime}$ corresponds to $\sim 199 h_{70}^{-1} \mathrm{kpc}$ at the cluster redshift.

\section{New optical data}

\subsection{Spectroscopic data}

Multi-object spectroscopic observations of A520 were carried out at the TNG telescope in December 2006. We used DOLORES/MOS with the LR-B Grism 1, yielding a dispersion of $187 \AA / \mathrm{mm}$, and the Loral CCD of $2048 \times 2048$ pixels (pixel size of $15 \mu \mathrm{m}$ ). This combination of grating and detector results in a dispersion of $2.8 \AA /$ pix. We observed three MOS masks for a total of 102 slits. We acquired three exposures of $1800 \mathrm{~s}$ for each mask. Wavelength calibration was performed using helium-argon lamps. Reduction of spectroscopic data was carried out with the IRAF ${ }^{2}$ package.

Radial velocities were determined using the crosscorrelation technique (Tonry \& Davis 1979) implemented in the RVSAO package (developed at the Smithsonian Astrophysical Observatory Telescope Data Center). Each spectrum was correlated against six templates for a variety of galaxy spectral types: E, S0, Sa, Sb, Sc, Ir (Kennicutt 1992). The template producing the highest value of $\mathcal{R}$, i.e., the parameter given by RVSAO and related to the signal-to-noise of the correlation peak, was chosen. Moreover, all spectra and their best correlation functions were examined visually to verify the redshift determination. The median value of $\mathcal{R}$ of our successfully measured galaxy redshifts is $\sim 8$. In nine cases (IDs 82, 86, 87, 143, 147 (QSO), 203, 229, 242 and 252; see Table 1) we took the EMSAO redshift as a reliable estimate of the redshift. Our spectroscopic survey in the field of A520 consists of 86 spectra with a median nominal error on $c z$ of $60 \mathrm{~km} \mathrm{~s}^{-1}$. The nominal errors as given by the cross-correlation are known to be smaller than the true errors (e.g. Malumuth et al. 1992; Bardelli et al. 1994; Ellingson \& Yee 1994; Quintana et al. 2000). Double redshift determinations for the same galaxy allowed us to estimate real intrinsic errors in data of the same quality taken with the same instrument (Barrena et al. 2007a,b). Here we applied a similar correction to our nominal errors, i.e. hereafter we assume that true errors are larger than nominal cross-correlation errors by a factor 1.5. Thus the median error on $c z$ is $90 \mathrm{~km} \mathrm{~s}^{-1}$.

\subsection{Photometric data}

As far as photometry is concerned, our observations were carried out with the Wide Field Camera (WFC), mounted at the prime focus of the $2.5 \mathrm{~m}$ INT telescope (located at Roque de los Muchachos observatory, La Palma, Spain). We observed A520 in January 2008 in photometric conditions and with a seeing of about 2 arcsec.

The WFC consists of a 4 chips mosaic covering a $30 \times 30$ arcmin field of view, with only a $20 \%$ marginally vignetted area. We took 15 exposures of $360 \mathrm{~s}$ using the $r$-SDSS $\left(r^{\prime}\right)$ filter, completing a total of $5400 \mathrm{~s}$ in this band. Moreover, we developed a dithering pattern in order to build a master "supersky" image that was used to correct our images for fringing patterns (Gullixson 1992). In addition, the dithering helped us to clean cosmic rays and avoid gaps between CCD chips. The complete reduction process (including flat fielding, bias subtraction and bad columns elimination) yielded a final co-added image where the variation of the sky was lower than $1 \%$ in the whole frame.

Another effect associated with the wide field frames is the distortion of the field. In order to match the photometric and spectroscopic samples, a good astrometric solution taking into account these distortions is needed. Using IRAF tasks and taking as reference the USNO B1.0 catalog we were able to find an accurate astrometric solution ( $\mathrm{rms} \sim 0.3 \mathrm{arcsec}$ ) across the full frame. The photometric calibration was performed using Landolt standard fields with well known $r^{\prime}$ magnitude. These fields were achieved during the observation. We finally identified galaxies in our image and measured their magnitudes with the SExtractor package (Bertin \& Arnouts 1996) and AUTOMAG procedure.

\footnotetext{
2 IRAF is distributed by the National Optical Astronomy Observatories, which are operated by the Association of Universities for Research in Astronomy, Inc., under cooperative agreement with the National Science Foundation.
} 
Table 1. Velocity catalog of 293 spectroscopically measured galaxies in the field of A520. In Col. 1, IDs in italics indicate non-cluster galaxies.

\begin{tabular}{|c|c|c|c|c|c|c|c|c|c|c|c|}
\hline \multirow{3}{*}{$\overline{\mathrm{ID}}$} & \multirow{3}{*}{$\alpha, \delta(\mathrm{J} 2000)$} & \multirow{3}{*}{$r^{\prime}$} & \multirow{3}{*}{$\begin{array}{c}v \\
\left(\mathrm{~km} \mathrm{~s}^{-1}\right)\end{array}$} & \multirow{3}{*}{$\begin{array}{c}\Delta v \\
\left(\mathrm{~km} \mathrm{~s}^{-1}\right)\end{array}$} & \multirow{3}{*}{ Source } & ID & $\alpha, \delta(\mathrm{J} 2000)$ & $r^{\prime}$ & & & Source \\
\hline & & & & & & & & & $\left(\mathrm{km} \mathrm{s}^{-1}\right)$ & $\left(\mathrm{km} \mathrm{s}^{-1}\right)$ & \\
\hline & & & & & & 71 & $045352.22,+025932.5$ & 21.78 & 65364 & 117 & $\mathrm{C}$ \\
\hline 1 & $045248.06,+030043.2$ & 19.62 & 94953 & 99 & $\mathrm{C}$ & 72 & $045352.44,+025414.7$ & 19.91 & 65804 & 58 & $\mathrm{~T}$ \\
\hline 2 & $045250.27,+025724.7$ & 20.13 & 68383 & 153 & $\mathrm{C}$ & 73 & $045352.53,+025432.7$ & 19.57 & 62582 & 130 & $\mathrm{C}$ \\
\hline 3 & $045250.93,+025437.9$ & 20.87 & 60186 & 140 & C & 74 & $045353.31,+025504.5$ & 20.59 & 115810 & 225 & $\mathrm{C}$ \\
\hline 4 & $045251.30,+025801.7$ & 20.01 & $\begin{array}{l}00180 \\
98925\end{array}$ & $\begin{array}{l}140 \\
104\end{array}$ & C & 75 & $045353.46,+025212.1$ & 18.86 & 67466 & 102 & $\mathrm{~T}$ \\
\hline $\begin{array}{l}4 \\
5\end{array}$ & $045251.91,+025644.9$ & $\begin{array}{l}20.21 \\
1950\end{array}$ & 98922 & 104 & $\mathrm{C}$ & 76 & $045354.33,+025639.2$ & 19.29 & 61182 & 126 & $\mathrm{C}$ \\
\hline$\frac{5}{6}$ & & 19.50 & 68173 & 112 & $\mathrm{C}$ & 77 & $045355.31,+025556.5$ & 18.26 & 60198 & 122 & $\mathrm{C}$ \\
\hline 6 & & 19.75 & 108794 & 225 & $\mathrm{C}$ & 78 & $045355.74,+025746.6$ & 18.83 & 59254 & 126 & $\mathrm{C}$ \\
\hline 7 & $045252.60,+025821.2$ & 19.68 & 56568 & 90 & $\mathrm{C}$ & 79 & $045355.99,+024845.7$ & 19.21 & 61134 & 60 & $\mathrm{~T}$ \\
\hline 8 & & 19.89 & 60738 & 117 & $\mathrm{C}$ & 80 & $045357.31,+025254.4$ & 19.44 & 62808 & 135 & $\mathrm{~T}$ \\
\hline 9 & $045254.83,+025854.2$ & 21.32 & 103989 & 225 & $\mathrm{C}$ & 81 & $045358.20,+030037.8$ & 19.49 & 59476 & 140 & $\mathrm{C}$ \\
\hline 10 & $045257.91,+025457.3$ & 18.25 & 60918 & 108 & $\mathrm{C}$ & 82 & $045358.33,+024718.1$ & 19.47 & 115868 & 64 & $\mathrm{~T}$ \\
\hline 11 & $045258.01,+025916.8$ & 19.66 & 54316 & 126 & $\mathrm{C}$ & 83 & $045358.35,+025830.5$ & 19.54 & 60492 & 748 & $\mathrm{C}$ \\
\hline 12 & $045258.76,+030002.7$ & 20.83 & 90780 & 94 & $\mathrm{C}$ & 84 & $045358.54,+024945.0$ & 19.10 & 65115 & 87 & $\mathrm{~T}$ \\
\hline 13 & $045259.62,+025734.1$ & 19.89 & 88858 & 112 & $\mathrm{C}$ & 85 & $045358.83,+025933.4$ & 19.34 & 60357 & 99 & $\mathrm{C}$ \\
\hline 14 & $045300.24,+025753.3$ & 20.92 & 99564 & 90 & $\mathrm{C}$ & 86 & $045359.04,+025057.3$ & 19.67 & 64947 & 27 & $\mathrm{~T}$ \\
\hline 15 & $045304.06,+025609.4$ & 20.61 & 59293 & 130 & $\mathrm{C}$ & 87 & $045359.29,+025307.2$ & 20.41 & 76130 & 21 & $\mathrm{~T}$ \\
\hline 16 & $045305.59,+025604.1$ & 19.99 & 64479 & 126 & $\mathrm{C}$ & 88 & $045359.36,+025117.1$ & 19.45 & 76190 & 63 & $\mathrm{~T}$ \\
\hline 17 & $045305.61,+030035.1$ & 20.16 & 60606 & 99 & $\mathrm{C}$ & 89 & $045359.59,+025636.5$ & 20.04 & 62429 & 148 & $\mathrm{C}$ \\
\hline 18 & $045307.50,+025743.4$ & 20.61 & 116442 & 225 & $\mathrm{C}$ & 90 & $045359.99,+025945.1$ & 18.40 & 49274 & 117 & $\mathrm{C}$ \\
\hline 19 & $045310.30,+030012.7$ & 20.98 & 91469 & 135 & $\mathrm{C}$ & 91 & $045400.34,+030355.9$ & 19.39 & 70008 & 80 & $\mathrm{~T}$ \\
\hline 20 & $045312.90,+025543.5$ & 20.84 & 77805 & 135 & $\mathrm{C}$ & 92 & $045400.56,+025332.9$ & 19.58 & 61319 & 144 & $\mathrm{C}$ \\
\hline 21 & $045313.68,+025505.3$ & 20.58 & 59383 & 117 & $\mathrm{C}$ & 93 & $045400.73,+024819.2$ & 19.66 & 64849 & 88 & $\mathrm{~T}$ \\
\hline 22 & $045314.19,+025552.0$ & 19.39 & 61964 & 108 & $\mathrm{C}$ & 94 & $045400.88,+025916.5$ & 20.62 & 77280 & 135 & $\mathrm{C}$ \\
\hline 23 & $045315.37,+025419.6$ & 19.90 & 64440 & 162 & C & 95 & $045401.15,+025745.6$ & 17.35 & 62154 & 51 & $\mathrm{C}+\mathrm{P}$ \\
\hline 24 & $045316.47,+025647.3$ & 20.89 & 98509 & 126 & $\mathrm{C}$ & 96 & $045401.76,+025547.4$ & 19.17 & 58327 & 90 & $\mathrm{C}$ \\
\hline 25 & $045317.28,+025515.1$ & 19.72 & 77751 & 112 & $\mathrm{C}$ & 97 & $045402.37,+030158.2$ & 19.34 & 59542 & 68 & $\mathrm{~T}$ \\
\hline 26 & $045320.43,+025805.0$ & 19.90 & 60741 & 130 & $\mathrm{C}$ & 98 & $045402.49,+025931.0$ & 19.25 & 49364 & 320 & $\mathrm{C}$ \\
\hline 27 & $045322.74,+025559.6$ & 18.25 & 61547 & 108 & $\mathrm{C}$ & 99 & $045402.71,+025036.2$ & 19.61 & 58860 & 68 & $\mathrm{~T}$ \\
\hline 28 & $045322.83,+025545.4$ & 19.77 & 62471 & 99 & $\mathrm{C}$ & 100 & $045402.88,+025222.6$ & 18.63 & 60818 & 76 & $\mathrm{~T}$ \\
\hline 29 & $045323.03,+025918.6$ & 18.62 & 59722 & 104 & $\mathrm{C}$ & 101 & $045402.90,+025106.7$ & 19.61 & 60461 & 116 & $\mathrm{~T}$ \\
\hline 30 & $045323.35,+025734.8$ & 18.79 & 62225 & 112 & $\mathrm{C}$ & 102 & $045403.05,+024934.7$ & 20.24 & 58347 & 117 & $\mathrm{~T}$ \\
\hline 31 & $045323.95,+025833.0$ & 19.74 & 60495 & 130 & $\mathrm{C}$ & 103 & $045403.26,+030440.5$ & 20.83 & 152875 & 112 & $\mathrm{~T}$ \\
\hline 32 & $045324.99,+025746.2$ & 19.64 & 78258 & 126 & $\mathrm{C}$ & 104 & $045403.36,+025540.3$ & 19.74 & 70034 & 144 & $\mathrm{C}$ \\
\hline 33 & $045325.04,+030025.7$ & 19.48 & 60057 & 126 & $\mathrm{C}$ & 105 & $045403.45,+025930.6$ & 18.79 & 60516 & 122 & $\mathrm{C}$ \\
\hline 34 & $045326.03,+025636.7$ & 20.21 & 98524 & 130 & $\mathrm{C}$ & 106 & $045403.82,+025332.4$ & 17.10 & 61277 & 114 & $\mathrm{~T}$ \\
\hline 35 & $045326.11,+025746.7$ & 18.70 & 99015 & 122 & $\mathrm{C}$ & 107 & $045403.96,+025340.7$ & 17.98 & 64646 & 70 & $\mathrm{~T}+\mathrm{C}$ \\
\hline 36 & $045327.14,+025738.8$ & 21.22 & 139946 & 225 & $\mathrm{C}$ & 108 & $045404.18,+030248.4$ & 20.84 & 55511 & 104 & $\mathrm{~T}$ \\
\hline 37 & $045329.33,+025658.9$ & 19.22 & 60729 & 99 & $\mathrm{C}$ & 109 & $045404.30,+024900.8$ & 20.01 & 59783 & 141 & $\mathrm{~T}$ \\
\hline 38 & $045329.61,+030031.8$ & 19.66 & 58588 & 86 & $\mathrm{C}$ & 110 & $045404.54,+025243.4$ & 19.08 & 60827 & 98 & $\mathrm{~T}$ \\
\hline 39 & $045331.36,+025509.9$ & 20.08 & 79496 & 130 & $\mathrm{C}$ & 111 & $045404.59,+025654.2$ & 20.12 & 61145 & 112 & $\mathrm{P}$ \\
\hline 40 & $045331.79,+025832.1$ & 20.76 & 69786 & 126 & $\mathrm{C}$ & 112 & $045404.67,+025604.0$ & 20.18 & 60327 & 148 & $\mathrm{C}$ \\
\hline 41 & $045332.65,+025553.7$ & 18.14 & 36736 & 112 & $\mathrm{C}$ & 113 & $045405.13,+024709.1$ & 19.26 & 65020 & 80 & $\mathrm{~T}$ \\
\hline 42 & $045333.63,+025457.9$ & 19.61 & 63283 & 140 & $\mathrm{C}$ & 114 & $045405.14,+025622.2$ & 20.02 & 59536 & 130 & $\mathrm{C}$ \\
\hline 43 & $045334.62,+025632.4$ & 19.58 & 59880 & 99 & $\mathrm{C}$ & 115 & $045405.38,+030431.0$ & 20.26 & 70903 & 92 & $\mathrm{~T}$ \\
\hline 44 & $045335.76,+025831.5$ & 17.29 & 59488 & 122 & $\mathrm{C}$ & 116 & $045405.44,+025915.5$ & 19.76 & 66554 & 117 & $\mathrm{C}$ \\
\hline 45 & $045336.05,+025503.4$ & 20.86 & 59353 & 126 & $\mathrm{C}$ & 117 & $045405.92,+025554.2$ & 19.92 & 59916 & 72 & $\mathrm{C}+\mathrm{P}$ \\
\hline 46 & $045336.54,+030001.6$ & 21.03 & 59401 & 144 & $\mathrm{C}$ & 118 & $045405.92,+025546.0$ & 20.15 & 58669 & 108 & $\mathrm{C}$ \\
\hline 47 & $045336.76,+025637.3$ & 18.85 & 64659 & 117 & $\mathrm{C}$ & 119 & $045405.93,+025337.3$ & 19.82 & 59458 & 153 & $\mathrm{C}$ \\
\hline 48 & $045336.99,+025747.3$ & 19.84 & 59320 & 99 & $\mathrm{C}$ & 120 & $045406.02,+025756.7$ & 21.22 & 62839 & 104 & $\mathrm{C}$ \\
\hline 49 & $045337.01,+025448.7$ & 20.90 & 79073 & 104 & $\mathrm{C}$ & 121 & $045406.12,+025846.1$ & 20.20 & 62018 & 450 & $\mathrm{C}$ \\
\hline 50 & $045338.36,+025731.6$ & 19.88 & 59272 & 130 & $\mathrm{C}$ & 122 & $045406.32,+030327.9$ & 19.67 & 59639 & 128 & $\mathrm{~T}$ \\
\hline 51 & $045339.06,+025710.3$ & 18.99 & 59707 & 112 & $\mathrm{C}$ & 123 & $045406.64,+025906.2$ & 20.09 & 98850 & 122 & $\mathrm{C}$ \\
\hline 52 & $045341.08,+025809.1$ & 20.85 & 62576 & 94 & $\mathrm{C}$ & 124 & $045406.77,+025355.4$ & 19.59 & 61473 & 71 & $\mathrm{~T}+\mathrm{C}$ \\
\hline 53 & $045341.56,+025523.4$ & 18.18 & 62962 & 108 & $\mathrm{C}$ & 125 & $045406.78,+025741.6$ & 20.39 & 66990 & 54 & $\mathrm{C}+\mathrm{P}$ \\
\hline 54 & $045341.88,+025729.9$ & 19.84 & 59832 & 135 & $\mathrm{C}$ & 126 & $045406.82,+030356.0$ & 19.38 & 70420 & 64 & $\mathrm{~T}$ \\
\hline 55 & 0453 41.97, +02 5900.3 & 19.26 & 60168 & 130 & $\mathrm{C}$ & 127 & $045407.47,+025144.6$ & 20.70 & 59358 & 93 & $\mathrm{~T}$ \\
\hline 56 & $045342.44,+025509.4$ & 19.68 & 58241 & 126 & $\mathrm{C}$ & 128 & $045407.62,+030059.2$ & 19.31 & 59490 & 46 & $\mathrm{~T}+\mathrm{C}$ \\
\hline 57 & $045342.56,+025733.9$ & 18.73 & 64665 & 122 & $\mathrm{C}$ & 129 & $045407.74,+025600.4$ & 19.78 & 60861 & 104 & $\mathrm{C}$ \\
\hline 58 & $045342.86,+025958.9$ & 18.55 & 44786 & 126 & $\mathrm{C}$ & 130 & $045407.83,+025702.7$ & 19.82 & 61140 & 122 & $\mathrm{C}$ \\
\hline 59 & $045342.89,+025419.3$ & 20.37 & 58729 & 117 & $\mathrm{C}$ & 131 & $045408.47,+025908.4$ & 20.03 & 60060 & 117 & $\mathrm{C}$ \\
\hline 60 & $045343.19,+030004.2$ & 20.18 & 98703 & 158 & $\mathrm{C}$ & 132 & $045408.63,+025636.1$ & 19.19 & 62695 & 94 & $\mathrm{C}$ \\
\hline 61 & $045343.32,+025859.9$ & 18.67 & 65472 & 94 & $\mathrm{C}$ & 133 & $045408.78,+030121.4$ & 19.10 & 60931 & 72 & $\mathrm{~T}$ \\
\hline 62 & $045343.78,+025922.2$ & 20.96 & 79478 & 94 & $\mathrm{C}$ & 134 & $045408.87,+025349.5$ & 18.69 & 57872 & 117 & $\mathrm{C}$ \\
\hline 63 & $045343.81,+025612.9$ & 18.65 & 65247 & 130 & $\mathrm{C}$ & 135 & $045408.90,+025321.5$ & 19.11 & 59219 & 66 & $\mathrm{~T}$ \\
\hline 64 & $045346.26,+025645.8$ & 18.68 & 64569 & 108 & $\mathrm{C}$ & 136 & $045409.03,+025200.8$ & 18.63 & 63622 & 66 & $\mathrm{~T}$ \\
\hline 65 & $045346.77,+025419.9$ & 20.07 & 61199 & 158 & $\mathrm{C}$ & 137 & $045409.06,+025948.8$ & 17.93 & 60075 & 76 & $\mathrm{C}$ \\
\hline 66 & $045347.93,+025730.2$ & 20.05 & 59578 & 117 & $\mathrm{C}$ & 138 & $045409.37,+025515.8$ & 18.97 & 61125 & 117 & $\mathrm{C}$ \\
\hline 67 & $045349.09,+025550.5$ & 18.97 & 75509 & 108 & $\mathrm{C}$ & 139 & $045409.41,+025022.7$ & 19.66 & 60440 & 104 & $\mathrm{~T}$ \\
\hline 68 & $045349.45,+025650.2$ & 20.27 & 65478 & 126 & $\mathrm{C}$ & 140 & $045409.41,+025132.1$ & 19.38 & 61024 & 48 & $\mathrm{~T}$ \\
\hline 69 & $045350.05,+025502.7$ & 18.64 & 59467 & 135 & $\mathrm{C}$ & 141 & $045409.42,+025626.3$ & 19.89 & 67036 & 94 & $\mathrm{C}$ \\
\hline 70 & $045350.16,+025710.2$ & 19.60 & 61436 & 122 & $\mathrm{C}$ & 142 & $045409.55,+025540.5$ & 19.20 & 58879 & 112 & $\mathrm{C}$ \\
\hline
\end{tabular}


Table 1. continued.

Table 1. continued.

\begin{tabular}{|c|c|c|c|c|c|c|c|c|c|c|c|}
\hline ID & $\alpha, \delta(\mathrm{J} 2000)$ & $r^{\prime}$ & $\begin{array}{c}v \\
\left(\mathrm{~km} \mathrm{~s}^{-1}\right)\end{array}$ & $\begin{array}{c}\Delta v \\
\left(\mathrm{~km} \mathrm{~s}^{-1}\right)\end{array}$ & Source & ID & $\overline{\alpha \alpha, \delta(\mathrm{J} 2000)}$ & $r^{\prime}$ & $\begin{array}{c}v \\
\left(\mathrm{~km} \mathrm{~s}^{-1}\right) \\
\end{array}$ & $\begin{array}{c}\Delta v \\
\left(\mathrm{~km} \mathrm{~s}^{-1}\right)\end{array}$ & Source \\
\hline 143 & $045409.60,+030222.9$ & 20.83 & 181270 & 51 & $\mathrm{~T}$ & 213 & $045421.72,+025556.0$ & 20.03 & 58244 & 140 & $\mathrm{C}$ \\
\hline 144 & $045410.10,+025542.2$ & 19.69 & 60600 & 117 & $\mathrm{C}$ & 214 & $045421.73,+030511.6$ & 19.79 & 61250 & 74 & $\mathrm{~T}$ \\
\hline 145 & $045410.31,+025438.9$ & 19.41 & 60963 & 51 & $\mathrm{~T}+\mathrm{C}$ & 215 & $045421.84,+025500.0$ & 19.78 & 58982 & 100 & $\mathrm{~T}$ \\
\hline 146 & $045410.41+025609.9$ & 20.02 & 58280 & 148 & $\mathrm{~T}+\mathrm{C}$ & 216 & $045422.02,+025743.3$ & 22.27 & 110602 & 225 & $\mathrm{C}$ \\
\hline 147 & $045410.52,+024739.2$ & 19.83 & 704502 & $\begin{array}{l}148 \\
100\end{array}$ & C & 217 & $045423.08,+025018.9$ & 20.93 & 165148 & 88 & $\mathrm{~T}$ \\
\hline 148 & $045410.69+030220.0$ & 20.11 & 152100 & 100 & $\mathrm{~T}$ & 218 & $045423.13,+025801.2$ & 20.03 & 60228 & 130 & $\mathrm{C}$ \\
\hline 149 & $045411.48,+025525.8$ & 20.26 & $\begin{array}{r}57941 \\
5700\end{array}$ & 96 & $\mathrm{~T}$ & 219 & $045423.27,+025709.0$ &.-- & 17085 & 108 & $\mathrm{C}$ \\
\hline 150 & $045411.69,+025913.1$ & 19.98 & 62291 & 108 & $\mathrm{C}$ & 220 & $045423.27,+025913.0$ & 19.83 & 61202 & 94 & $\mathrm{C}$ \\
\hline 151 & $045411.79,+024810.7$ & 18.28 & 58729 & $\begin{array}{r}135 \\
56\end{array}$ & $\mathrm{C}$ & 221 & $045423.48,+030316.1$ & 20.94 & 78236 & 238 & $\mathrm{~T}$ \\
\hline 152 & $045411.80,+025211.4$ & 19.60 & 60376 & $\begin{array}{l}56 \\
54\end{array}$ & $\mathrm{~T}$ & 222 & $045423.53,+025034.5$ & 18.50 & 60667 & 69 & $\mathrm{~T}$ \\
\hline 153 & $045411.82,+025048.1$ & 19.11 & 59577 & $\begin{array}{r}54 \\
182\end{array}$ & $\mathrm{~T}$ & 223 & $045423.84,+025108.6$ & 20.14 & 114162 & 98 & $\mathrm{~T}$ \\
\hline 154 & $045411.93,+025807.8$ & 18.23 & 62292 & $\begin{array}{r}182 \\
47\end{array}$ & $\mathrm{~T}$ & 224 & $045423.99,+025610.9$ & 21.16 & 70466 & 108 & $\mathrm{C}$ \\
\hline 155 & $045412.08,+025636.5$ & 20.77 & 60708 & $\begin{array}{r}47 \\
148\end{array}$ & $\mathrm{C}+\mathrm{P}$ & 225 & $045424.24,+030111.9$ & 21.50 & 79751 & 117 & $\mathrm{~T}$ \\
\hline 156 & $045412.19,+025750.7$ & 20.48 & 58417 & $\begin{array}{l}148 \\
153\end{array}$ & $\mathrm{C}$ & 226 & $045424.88,+025856.0$ & 19.93 & 60289 & 87 & $\mathrm{C}+\mathrm{P}$ \\
\hline 157 & $045412.31,+030247.8$ & 20.89 & 85271 & $\begin{array}{l}153 \\
188\end{array}$ & $\mathrm{C}$ & 227 & $045424.95,+025223.5$ & 20.02 & 60260 & 90 & $\mathrm{~T}$ \\
\hline 158 & $045412.77,+024956.5$ & 19.66 & 82005 & $\begin{array}{r}188 \\
78\end{array}$ & $\mathrm{~T}$ & 228 & $045425.34,+024703.9$ & 19.70 & 61235 & 72 & $\mathrm{~T}$ \\
\hline 159 & $045413.04,+025633.2$ & 19.36 & 60084 & 144 & C & 229 & $045425.37,+024923.4$ & 20.62 & 57854 & 226 & $\mathrm{~T}$ \\
\hline 160 & $045413.14,+025733.8$ & 17.70 & 60115 & 24 & P & 230 & $045425.50,+025938.3$ & 18.98 & 60057 & 104 & $\mathrm{C}$ \\
\hline 161 & $045413.16,+025836.6$ & 20.25 & 62495 & 135 & $\mathrm{C}$ & 231 & $045426.18,+025423.3$ & 21.25 & 50350 & 135 & $\mathrm{C}$ \\
\hline 162 & $045413.34,+030208.8$ & 20.65 & 99114 & 106 & $\mathrm{~T}$ & 232 & $045426.63,+030046.9$ & 19.87 & 60182 & 77 & $\mathrm{~T}+\mathrm{C}$ \\
\hline 163 & $045413.35,+025158.1$ & 20.05 & 60776 & 96 & $\mathrm{~T}$ & 233 & $045426.80,+025821.9$ & 19.86 & 78677 & 135 & $\mathrm{C}$ \\
\hline 164 & $045413.50,+024833.7$ & 20.45 & 75930 & 180 & $\mathrm{~T}$ & 234 & $045427.64,+030329.3$ & 20.02 & 60186 & 99 & $\mathrm{~T}$ \\
\hline 165 & $045413.68,+025610.2$ & 19.78 & 59653 & 99 & $\mathrm{C}$ & 235 & $045427.76,+025529.2$ & 18.63 & 67171 & 90 & $\mathrm{C}$ \\
\hline 166 & $045413.74,+025326.7$ & 19.14 & 60519 & 72 & $\mathrm{~T}$ & 236 & 0454 27.96, +02 5418.1 & 19.61 & 61070 & 93 & $\mathrm{~T}$ \\
\hline 167 & $045413.80,+025919.4$ & 19.81 & 59059 & 130 & $\mathrm{C}$ & 237 & $045428.14,+025545.7$ & 20.41 & 59611 & 112 & $\mathrm{C}$ \\
\hline 168 & $045414.01,+025542.5$ & 19.63 & 59383 & 122 & $\mathrm{C}$ & 238 & $045428.18,+025536.5$ & 18.74 & 66893 & 99 & $\mathrm{C}$ \\
\hline 169 & $045414.09,+030105.1$ & 20.39 & 40586 & 144 & $\mathrm{C}$ & 239 & $045428.63,+030416.0$ & 19.17 & 60508 & 62 & $\mathrm{~T}$ \\
\hline 170 & $045414.10,+025709.9$ & 17.29 & 59506 & 69 & $\mathrm{P}$ & 240 & $045429.02,+025429.0$ & 19.97 & 65186 & 61 & $\mathrm{~T}+\mathrm{C}$ \\
\hline 171 & $045414.17,+030110.3$ & 18.89 & 66961 & 140 & $\mathrm{C}$ & 241 & $045429.02,+025659.5$ & 20.54 & 62384 & 153 & $\mathrm{C}$ \\
\hline 172 & $045414.34,+025836.5$ & 18.50 & 60111 & 104 & $\mathrm{C}$ & 242 & $045429.14,+024855.9$ & 19.74 & 135190 & 366 & $\mathrm{~T}$ \\
\hline 173 & 0454 14.36, +02 5916.3 & 19.41 & 58513 & 117 & $\mathrm{C}$ & 243 & $045429.53,+025822.1$ & 19.34 & 67096 & 108 & $\mathrm{C}$ \\
\hline 174 & $045414.40,+025642.2$ & 19.02 & 60762 & 99 & $\mathrm{C}$ & 244 & $045429.58,+025522.0$ & 19.66 & 62063 & 112 & $\mathrm{C}$ \\
\hline 175 & $045414.79,+030049.0$ & 19.01 & 58513 & 75 & $\mathrm{~T}+\mathrm{C}$ & 245 & $045430.21,+030225.3$ & 18.59 & 66861 & 98 & $\mathrm{~T}$ \\
\hline 176 & $045415.09,+025707.8$ & 18.06 & 59163 & 80 & $\mathrm{C}+\mathrm{P}$ & 246 & $045430.31,+025844.8$ & 19.18 & 59877 & 104 & $\mathrm{C}$ \\
\hline 177 & $045415.55,+025458.3$ & 20.26 & 18338 & 270 & $\mathrm{C}$ & 247 & $045430.67,+025444.4$ & 20.05 & 66872 & 117 & $\mathrm{C}$ \\
\hline 178 & $045415.76,+025246.9$ & 19.09 & 62064 & 122 & $\mathrm{~T}$ & 248 & $045430.90,+025935.4$ & 19.93 & 17451 & 225 & $\mathrm{C}$ \\
\hline 179 & $045415.89,+030447.6$ & 20.62 & 58757 & 122 & $\mathrm{~T}$ & 249 & $045431.01,+024904.3$ & 20.05 & 60211 & 64 & $\mathrm{~T}$ \\
\hline 180 & $045415.95,+025819.1$ & 19.43 & 60267 & 135 & $\mathrm{C}$ & 250 & $045431.23,+030513.6$ & 18.97 & 60001 & 88 & $\mathrm{~T}$ \\
\hline 181 & $045416.01,+025520.7$ & 18.33 & 60954 & 104 & $\mathrm{C}$ & 251 & $045431.42,+025722.9$ & 20.36 & 74618 & 180 & $\mathrm{C}$ \\
\hline 182 & $045416.06,+025642.8$ & 18.64 & 58821 & 94 & $\mathrm{C}+\mathrm{P}$ & 252 & $045431.93,+025236.7$ & 20.39 & 76231 & 41 & $\mathrm{~T}+\mathrm{C}$ \\
\hline 183 & $045416.56,+025726.7$ & 19.67 & 58876 & 126 & $\mathrm{C}$ & 253 & $045432.31,+030352.1$ & 20.23 & 59672 & 147 & $\mathrm{~T}$ \\
\hline 184 & $045416.57,+025531.8$ & 19.35 & 60972 & 104 & $\mathrm{C}$ & 254 & $045432.63,+025301.3$ & 20.32 & 59221 & 164 & $\mathrm{~T}$ \\
\hline 185 & $045416.89,+025424.8$ & 20.25 & 62827 & 103 & $\mathrm{C}$ & 255 & $045432.68,+025448.9$ & 18.00 & 60894 & 59 & $\mathrm{C}+\mathrm{P}$ \\
\hline 186 & $045416.94,+024837.4$ & 20.03 & 82173 & 96 & $\mathrm{~T}$ & 256 & $045433.56,+030323.1$ & 19.10 & 99246 & 112 & $\mathrm{C}$ \\
\hline 187 & $045417.10,+030149.5$ & 19.35 & 60006 & 57 & $\mathrm{~T}$ & $\begin{array}{l}257 \\
258\end{array}$ & $045433.78,+025851.8$ & 20.91 & 125307 & 225 & $\mathrm{C}$ \\
\hline 188 & $045417.31,+025312.0$ & 19.07 & 60264 & 60 & $\mathrm{~T}$ & 258 & $045434.25,+025000.3$ & 19.46 & 111247 & 81 & $\mathrm{~T}$ \\
\hline 189 & $045417.33,+025646.1$ & 19.60 & 64056 & 122 & $\mathrm{P}$ & 259 & $045435.27,+030105.3$ & 18.84 & 59269 & 104 & $\mathrm{C}$ \\
\hline 190 & $045417.43,+025924.0$ & 19.54 & 58780 & 76 & $\mathrm{C}$ & 260 & $045435.83,+030105.4$ & 20.53 & 61005 & 153 & $\mathrm{C}$ \\
\hline 191 & $045417.66,+024824.9$ & 19.66 & 82097 & 78 & $\mathrm{~T}$ & 261 & $045436.49,+025433.7$ & 21.45 & 112128 & 225 & $\mathrm{C}$ \\
\hline 192 & $045417.90,+025535.0$ & 19.31 & 60549 & 117 & $\mathrm{C}$ & 262 & $045436.93,+030323.7$ & 19.63 & 60798 & 122 & $\mathrm{C}$ \\
\hline 193 & $045417.95,+024649.6$ & 18.94 & 60927 & 72 & $\mathrm{~T}$ & 263 & $045437.47,+030224.4$ & 18.65 & 60021 & 112 & $\mathrm{C}$ \\
\hline 194 & $045418.02,+025741.5$ & 20.14 & 61397 & 126 & $\mathrm{C}$ & 264 & $045438.54,+030051.5$ & 17.43 & 61074 & 104 & $\mathrm{C}$ \\
\hline 195 & $045418.18,+025955.7$ & 20.09 & 61334 & 130 & $\mathrm{C}$ & 265 & $045439.39,+030344.7$ & 20.44 & 60825 & 189 & $\mathrm{C}$ \\
\hline 196 & $045418.58,+030036.5$ & 20.45 & 57385 & 90 & $\mathrm{~T}$ & 266 & 0454 40.49, +02 5231.8 & 19.67 & 66941 & 144 & $\mathrm{C}$ \\
\hline 197 & $045418.88,+025054.4$ & 16.93 & 18754 & 100 & $\mathrm{~T}$ & 267 & $045440.76,+030235.9$ & 19.51 & 60006 & 117 & $\mathrm{C}$ \\
\hline 198 & $045419.00,+025617.2$ & 18.74 & 62504 & 135 & $\mathrm{C}$ & $\begin{array}{l}268 \\
269\end{array}$ & $045441.11,+025949.5$ & 21.97 & 113621 & 450 & $\mathrm{C}$ \\
\hline 199 & $045419.05,+025613.8$ & 20.94 & 91532 & 130 & $\mathrm{C}$ & 269 & $045441.67,+025917.8$ & 20.89 & 106558 & 108 & $\mathrm{C}$ \\
\hline 200 & $045419.16,+025826.5$ & 19.10 & 60552 & 117 & $\mathrm{C}$ & 270 & $045441.90,+025855.2$ & 19.60 & 69483 & 270 & $\mathrm{C}$ \\
\hline 201 & $045419.28,+030109.9$ & 20.64 & 58353 & 122 & $\mathrm{~T}+\mathrm{C}$ & 271 & $045442.25,+030202.1$ & 20.33 & 99066 & 126 & $\mathrm{C}$ \\
\hline 202 & $045419.31,+025147.5$ & 19.71 & 58948 & 60 & $\mathrm{~T}$ & 272 & $045442.63,+030158.3$ & 19.24 & 59602 & 144 & $\mathrm{C}$ \\
\hline 203 & $045419.51,+024805.7$ & 19.35 & 8555 & 31 & $\mathrm{~T}$ & 273 & $045443.01,+025742.3$ & 20.60 & 70781 & 72 & $\mathrm{C}$ \\
\hline 204 & $045419.91,+025744.8$ & 16.93 & 60315 & 64 & $\mathrm{C}+\mathrm{P}$ & 274 & 0454 44.96, +030106.2 & 20.78 & 113447 & 225 & $\mathrm{C}$ \\
\hline 205 & $045419.96,+025530.6$ & 17.35 & 58597 & 99 & $\mathrm{C}$ & 275 & $045445.34,+025340.4$ & 19.59 & 55623 & 104 & $\mathrm{C}$ \\
\hline 206 & $045420.17,+025532.5$ & 19.43 & 58381 & 108 & $\mathrm{C}$ & 276 & $045446.28,+025236.1$ & 20.56 & 77385 & 99 & $\mathrm{C}$ \\
\hline 207 & $045420.21,+025920.9$ & 20.88 & 59955 & 176 & $\mathrm{C}$ & 277 & $045446.85,+025321.9$ & 20.84 & 55396 & 99 & $\mathrm{C}$ \\
\hline 208 & $045420.56,+030055.9$ & 19.94 & 76010 & 123 & $\mathrm{~T}+\mathrm{C}$ & 278 & $045446.85,+030257.0$ & 21.49 & 98017 & 99 & $\mathrm{C}$ \\
\hline 209 & $045420.58,+025337.4$ & 19.52 & 59946 & 90 & $\mathrm{~T}+\mathrm{C}$ & 279 & $045447.88,+030333.3$ & 18.62 & 71557 & 112 & $\mathrm{C}$ \\
\hline 210 & $045420.62,+025641.4$ & 19.85 & 58657 & 81 & $\mathrm{C}$ & 280 & $045448.86,+025234.5$ & 20.66 & 62366 & 180 & $\mathrm{C}$ \\
\hline 211 & $045420.68,+025529.8$ & 18.32 & 58969 & 104 & $\mathrm{C}$ & 281 & 0454 49.21, +03 0323.4 & 20.20 & 99588 & 180 & $\mathrm{C}$ \\
\hline 212 & $045421.07,+025124.9$ & 19.08 & 61484 & 72 & $\mathrm{~T}$ & 282 & $045451.40,+025752.4$ & 19.39 & 45940 & 99 & $\mathrm{C}$ \\
\hline & & & & & & 283 & $045452.12,+025427.4$ & 20.81 & 69405 & 117 & $\mathrm{C}$ \\
\hline
\end{tabular}


Table 1. continued.

\begin{tabular}{|c|c|c|c|c|c|}
\hline \multirow[t]{2}{*}{ ID } & \multirow[t]{2}{*}{$\alpha, \delta(\mathrm{J} 2000)$} & \multirow[t]{2}{*}{$r^{\prime}$} & \multirow{2}{*}{$\begin{array}{c}v \\
\left(\mathrm{~km} \mathrm{~s}^{-1}\right)\end{array}$} & \multirow{2}{*}{$\begin{array}{c}\Delta v \\
\left(\mathrm{~km} \mathrm{~s}^{-1}\right)\end{array}$} & \multirow[t]{2}{*}{ Source } \\
\hline & & & & & \\
\hline 284 & $045452.32,+025642.7$ & 20.45 & 110251 & 225 & $\mathrm{C}$ \\
\hline 285 & $045453.87,+025245.2$ & 20.03 & 60537 & 126 & $\mathrm{C}$ \\
\hline 286 & $045455.00,+025400.0$ & 19.21 & 62489 & 148 & $\mathrm{C}$ \\
\hline 287 & $045455.47,+025831.7$ & 22.45 & 138135 & 144 & $\mathrm{C}$ \\
\hline 288 & $045456.66,+030053.4$ & 21.63 & 94455 & 112 & $\mathrm{C}$ \\
\hline 289 & $045456.87,+025402.9$ & 18.69 & 69321 & 99 & $\mathrm{C}$ \\
\hline 290 & $045457.20,+030326.6$ & 20.64 & 98625 & 104 & $\mathrm{C}$ \\
\hline 291 & $045458.37,+030154.0$ & 20.73 & 135512 & 225 & $\mathrm{C}$ \\
\hline 292 & $045459.89,+025331.1$ & 19.46 & 68559 & 135 & $\mathrm{C}$ \\
\hline 293 & $045501.66,+025755.7$ &.-- & 138036 & 225 & $\mathrm{C}$ \\
\hline
\end{tabular}

In few cases (e.g., close companion galaxies, galaxies close to defects of CCD), the standard SExtractor photometric procedure failed. In these cases we computed magnitudes by hand. This method consists in assuming a galaxy profile of a typical elliptical and scale it to the maximum observed value. The integration of this profile gives us an estimate of the magnitude. The idea of this method is similar to the PSF photometry, but assuming a galaxy profile, more appropriate in this case.

As a final step, we estimated and corrected the galactic extinction, $A_{r^{\prime}}=0.12$, from Burstein \& Heiles (1982) reddening maps. We estimated that our photometric sample is complete down to $r^{\prime}=22.0$ (23.0) for $S / N=5$ (3) within the observed field.

\section{Construction of the galaxy catalog}

In addition to our TNG data we considered redshifts coming from the CNOC survey (Carlberg et al. 1996; Yee et al. 1996). A detailed description of the data reduction techniques for the spectroscopic data is given in Yee et al. (1996). We considered the 215 galaxies having a redshift determined via a correlation significance parameter $\mathcal{R}^{\prime} \gtrsim 3$ as suggested by Yee et al. (1996), see also Ellingson \& Yee (1994) for the description of this parameter. As for TNG data, we applied the above correction to nominal errors leading to a median error on $c z$ of $\sim 120 \mathrm{~km} \mathrm{~s}^{-1}$. This error is quite in agreement with the error analysis performed by the CNOC authors (Ellingson \& Yee 1994).

Before we proceed with the merging between TNG and CNOC catalogs we payed particular attention to their compatibility. Twelve galaxies in the CNOC catalog are in common with our TNG catalog. Of these, one (galaxy ID 215) can be considered as strongly discrepant with a $\Delta c z$ difference of $\sim 1000 \mathrm{~km} \mathrm{~s}^{-1}\left[c z_{\mathrm{TNG}}=(58982 \pm 100) \mathrm{km} \mathrm{s}^{-1}\right.$ vs. $c z_{\mathrm{CNOC}}=$ $(59943 \pm 81) \mathrm{km} \mathrm{s}^{-1}$ ]. For this galaxy a redshift by Newberry et al. (1988) also exists and it is in agreement with the TNG redshift. For the remaining eleven galaxies we compared the TNG and CNOC determinations computing the mean and the rms of the variable $\left(z_{1}-z_{2}\right) / \sqrt{\operatorname{err}_{1}^{2}+\operatorname{err}_{2}^{2}}$, where $z_{1}$ comes from $\mathrm{TNG}$, and $z_{2}$ from CNOC. We obtained mean $=0.53 \pm 0.66$ and $\mathrm{rms}=2.2$, to be compared with the expected values of 0 and 1. The resulting mean shows that the two sets of measurements are consistent with having the same velocity zero-point according to the $\chi^{2}$-test. The high value of rms suggests that the errors are still underestimated. However, when rejecting another two slightly discrepant determinations $\left(\Delta c z \sim 700 \mathrm{~km} \mathrm{~s}^{-1}\right.$ for IDs 253 and 72) we obtained mean $=0.55 \pm 0.46$ and $\mathrm{rms}=1.4$, in good agreement with the expected values of 0 and 1 . We decided to take our TNG redshifts for the galaxies IDs 215, 253 and 72 and combine TNG and CNOC data using the weighted mean of the two redshift determinations and the corresponding errors for the remaining nine common galaxies. In total, we added another new 203 galaxies from CNOC obtaining a merged catalog of 289 galaxies.

Finally, we considered the catalog of galaxies in the field of A520 published by Proust et al. (2000, their Table 1). For three galaxies (the 2nd, 16th, and 19th) Proust et al. list only redshifts coming from old previous literature data. These galaxies are already present in our TNG catalog and we verified the agreement between our and previous redshift values. Out of the 24 galaxies measured by Proust et al., we considered only the 13 galaxies with $\mathcal{R} \gtrsim 3$ and one galaxy with redshift measured on the emission line $\mathrm{H} \alpha$. After having applied the correction to their nominal redshift, we checked the compatibility with our TNG+CNOC catalog using the method described above. We found nine galaxies in common with our catalog for which we obtain mean $=-0.23 \pm 0.46$ and $\mathrm{rms}=1.4$, in agreement with the expected values of 0 and 1 . We combined TNG+CNOC catalog and Proust et al. data using the weighted mean of the two redshift determinations and the corresponding error for the nine galaxies in common. We added another new four galaxies by Proust et al., two of which are very bright galaxies.

In summary, our redshift catalog of A520 consists of 293 galaxies sampling a wide, asymmetric cluster region (see Fig. 2) and having a median error on $c z$ of $112 \mathrm{~km} \mathrm{~s}^{-1}$. Table 1 lists the velocity catalog: identification number of each galaxy, ID (Col. 1); right ascension and declination, $\alpha$ and $\delta$ (J2000, Col. 2); $r^{\prime}$ magnitudes (Col. 3); heliocentric radial velocities, $v=c z_{\odot}(\mathrm{Col} .4)$ with errors, $\Delta v(\mathrm{Col} .5)$; redshift source (Col. 6; T: TNG, C: CNOC and P: Proust et al.). We list $r^{\prime}$ magnitudes for 291 out of 293 galaxies having redshifts. The exceptions are a galaxy just outside the western border of the imaging field and a huge foreground spiral galaxy. We have redshifts for galaxies down to $r^{\prime} \sim 21.5 \mathrm{mag}$, but we are $40 \%$ complete down to $r^{\prime}=19$ mag within 3 arcmin from RA $=04^{\mathrm{h}} 54^{\mathrm{m}} 14^{\mathrm{s}}$, Dec $=+02^{\circ} 57^{\prime} 00^{\prime \prime}(\mathrm{J} 2000.0)$. The completeness of the spectroscopic sample decreases in the outskirts of the cluster.

Figure 3 shows the contribution of TNG data added to previous spectroscopic information.

A520 does not exhibit the presence of a clear dominant galaxy and in fact it is classified as Bautz-Morgan class III (Abell et al. 1989). In particular, our sample lists nine luminous galaxies in a range of one mag from the most luminous one: IDs 204, $106,44,170,95,205,264,160$ and 137 . These galaxies are generally sparse in the field. A few of these galaxies are close to the lensing mass peaks pointed out by M07, i.e. ID 204 is close to peak No. 1; the galaxy couple composed by IDs 160 and 170 is close to peak No. 2; ID 106 is close to peak No. 4; ID 205 is close to peak No. 5.

Govoni et al. (2001b) pointed out the presence of several discrete radio sources in the field of A520. In particular, there are two head-tail radio sources $(0454+0255 \mathrm{~A}$ and $0454+0255 \mathrm{~B}$; see also Cooray et al. 1998) located on the eastern side with the tails oriented toward the same direction, opposite to the cluster center. Our catalog lists the redshift for the northern one, 0454+0255B (ID 184), which is classified as a cluster member. Cooray et al. (1998) also list a third radio source $(0454+0257)$ which, again, is classified as a cluster member (ID 95). From a visual inspection of the Chandra image studied by Markevitch et al. (2005, Obs.Id 4215) we also note that $0454+0257$ is an evident pointlike $X$-ray source in the field of A520. 


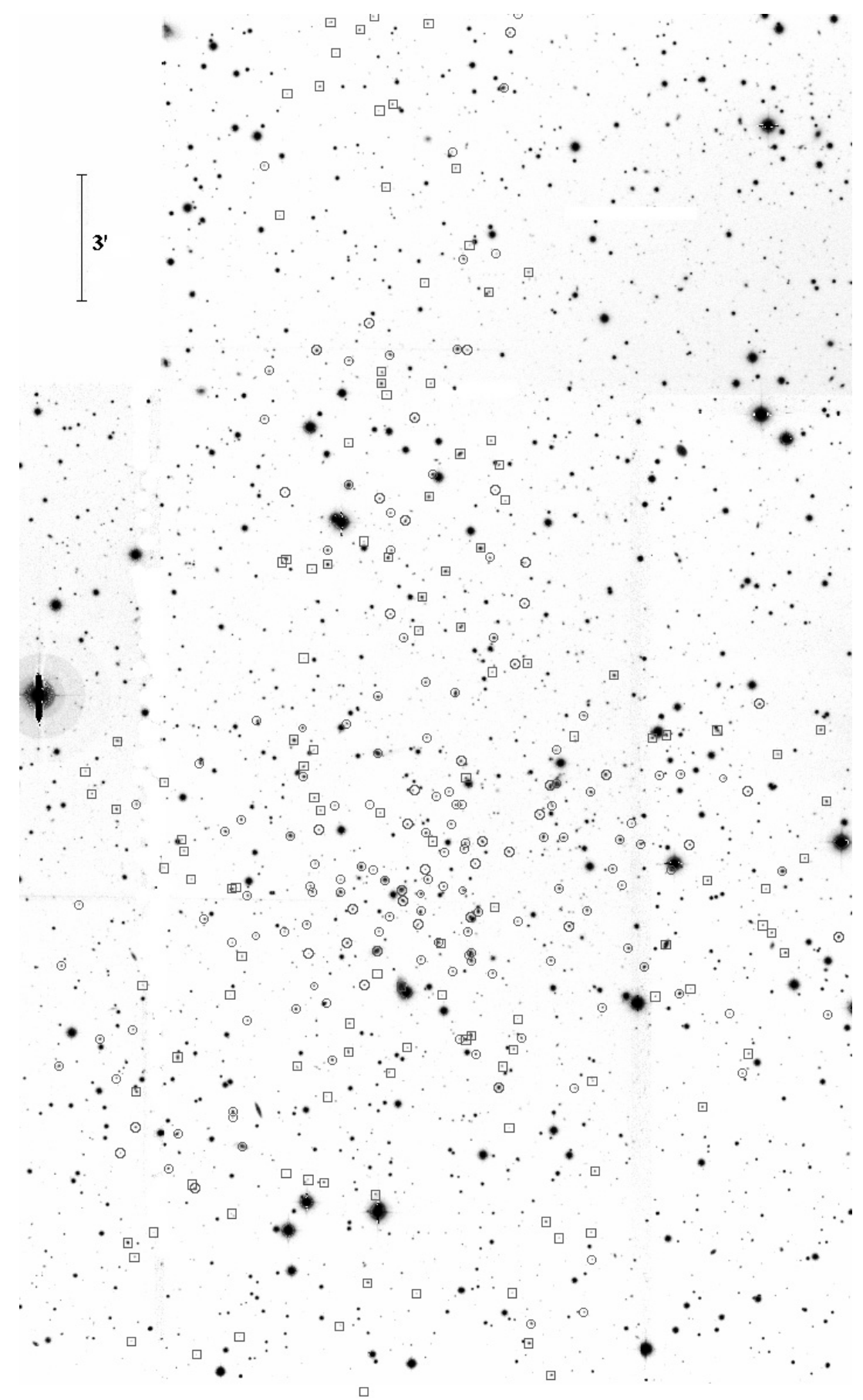

Fig. 2. INT $r^{\prime}$-band image of A520 (West at the top and North to the left). Circles and boxes indicate cluster members and non-member galaxies, respectively (see Table 1 ).

\section{Analysis and results}

\subsection{Member selection}

To select cluster members out of 293 galaxies having redshifts, we follow a two steps procedure. First, we perform the adaptive-kernel method (hereafter DEDICA, Pisani 1993 and 1996; see also Fadda et al. 1996; Girardi et al. 1996; Girardi $\&$ Mezzetti 2001). We search for significant peaks in the velocity distribution at $>99 \%$ c.1.. This procedure detects A520 as an asymmetric one-peak structure at $z \sim 0.201$ populated by 223 galaxies considered as candidate cluster members (see Fig. 4).

All the galaxies assigned to the A520 peak are analyzed in the second step which uses the combination of position and velocity information: the "shifting gapper" method by Fadda et al. (1996). This procedure rejects galaxies that are too far in velocity from the main body of galaxies and within a fixed bin that shifts along the distance from the cluster center. The procedure is iterated until the number of cluster members converges to a stable value. Following Fadda et al. (1996) we use a gap of $1000 \mathrm{~km} \mathrm{~s}^{-1}$ - in the cluster rest-frame - and a bin of $0.6 h_{70}^{-1} \mathrm{Mpc}$, or large enough to include 15 galaxies. The choice of the cluster center is not obvious. In fact, several galaxy condensations are visible in the field (Gioia \& Luppino 1994). Moreover, no obvious dominant galaxy is present (see Sect. 3) and the lensing mass distribution shows several peaks (e.g. M07). Thus, hereafter we assume the position of the peak 


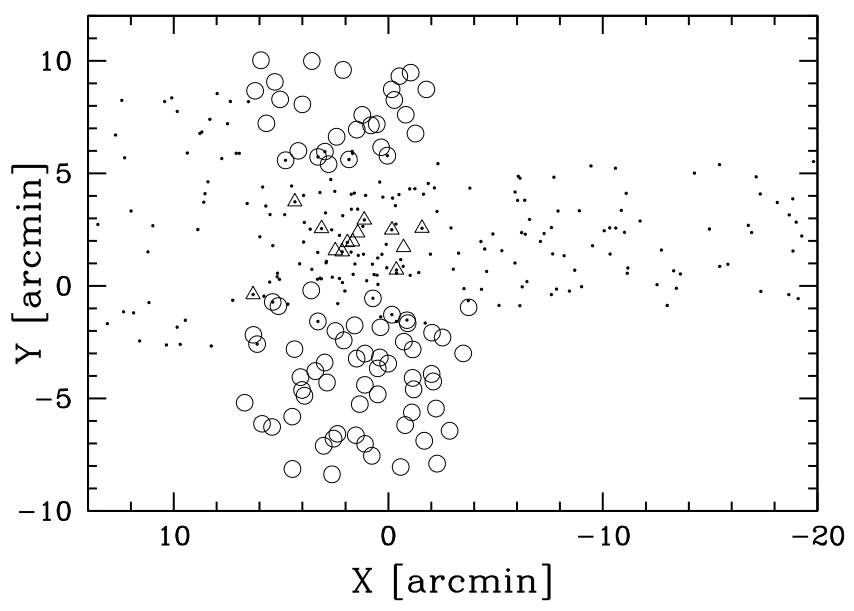

Fig. 3. Spatial distribution on the sky of the 293 galaxies having redshifts in the cluster field. Circles indicate galaxies having new redshifts acquired with the TNG. Dots and triangles indicate galaxies having redshift data from CNOC and Proust et al. (2000) catalogs, respectively. The X-ray peak is taken as the cluster center.

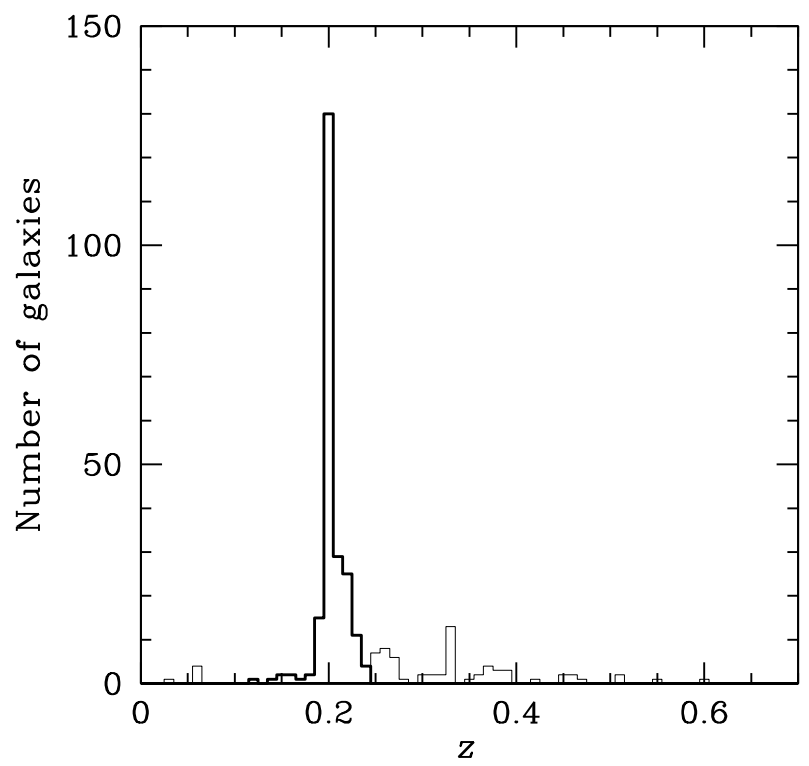

Fig. 4. Redshift galaxy distribution. The solid line histogram refers to the (223) galaxies assigned to the cluster according to the DEDICA reconstruction method.

of X-ray emission as listed by Ebeling et al. (1996) [RA = $04^{\mathrm{h}} 54^{\mathrm{m}} 07.44$, Dec $\left.=+02^{\circ} 55^{\prime} 12^{\prime \prime} .0(\mathrm{~J} 2000.0)\right]$ as the cluster center. After the "shifting gapper" procedure we obtain a sample of 167 fiducial cluster members (see Fig. 5).

The 2D galaxy distribution analyzed through the 2D DEDICA method shows only one peak [at RA $=04^{\mathrm{h}} 54^{\mathrm{m}} 13.55$, Dec. $\left.=+02^{\circ} 56^{\prime} 35^{\prime \prime 2}(\mathrm{~J} 2000.0)\right]$. This peak, hereafter the "optical" cluster center, is displaced towards NE with respect to the $\mathrm{X}$-ray peak and is close, but not coincident, to a pair of luminous galaxies (IDs 160 and 170). The biweight cluster center, i.e. that recovered by computing the biweight means (Beers et al. 1990) of RA and Dec. of galaxy positions $\left[\mathrm{RA}=04^{\mathrm{h}} 54^{\mathrm{m}} 12.62\right.$, Dec. $=+02^{\circ} 55^{\prime} 57$ !'3 (J2000.0)], is roughly coincident with the DEDICA peak. Using these alternative cluster centers we verify the robustness of our member selection.

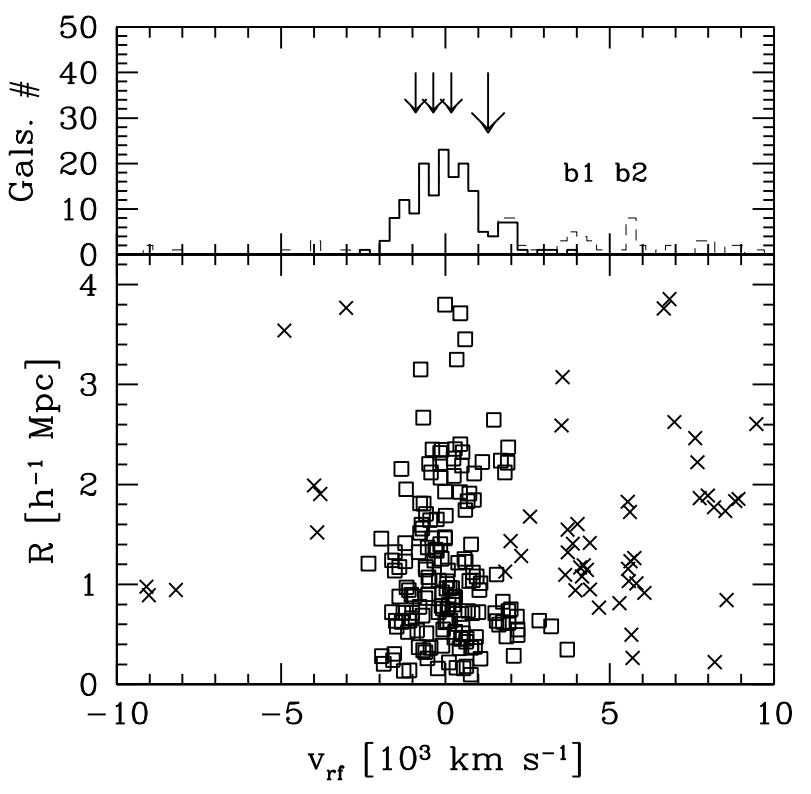

Fig. 5. Lower panel: projected clustercentric distance vs. rest-frame velocity for the 223 galaxies in the main peak (Fig. 4). Crosses show galaxies detected as interlopers by our "shifting gapper" procedure. Upper panel: rest-frame velocity histogram for the 223 galaxies in the main peak; the solid line refers to the 167 cluster members only. Large and small arrows indicate the positions of weighted gaps in the velocity distribution of the whole sample and of the main system (MS). Labels b1 and b2 indicate back1 and back2 "background" peaks of galaxies.

\subsection{Global kinematical properties}

By applying the biweight estimator to the 167 cluster members (Beers et al. 1990), we compute a mean cluster redshift of $\langle z\rangle=0.2008 \pm 0.0003$, i.e. $\langle v\rangle=(60209 \pm 82) \mathrm{km} \mathrm{s}^{-1}$. We estimate the LOS velocity dispersion, $\sigma_{v}$, by using the biweight estimator and applying the cosmological correction and the standard correction for velocity errors (Danese et al. 1980). We obtain $\sigma_{\mathrm{v}}=1066_{-61}^{+67} \mathrm{~km} \mathrm{~s}^{-1}$, where errors are estimated through a bootstrap technique.

To evaluate the robustness of the $\sigma_{\mathrm{v}}$ estimate we analyze the velocity dispersion profile (Fig. 6). The integral profile smoothly decreases and flattens beyond $\sim 0.6 h_{70}^{-1} \mathrm{Mpc}$ suggesting that a robust value of $\sigma_{\mathrm{v}}$ is asymptotically reached in the external cluster regions, as found for most nearby clusters (e.g. Fadda et al. 1996; Girardi et al. 1996).

\subsection{Substructure}

\subsubsection{Velocity distribution}

We analyze the velocity distribution to look for possible deviations from Gaussianity that might provide important signatures of complex dynamics. For the following tests the null hypothesis is that the velocity distribution is a single Gaussian.

We estimate three shape estimators, i.e. the kurtosis, the skewness, and the scaled tail index (see, e.g. Beers et al. 1991). According to the value of the skewness $(+0.471)$ the velocity distribution is positively skewed and differs from a Gaussian at the 95-99\% c.l. (see Table 2 of Bird \& Beers 1993). Moreover, according to the scaled tail index the velocity distribution is heavily tailed and differs from a Gaussian at the 90-95\% c.l. (see Table 2 of Bird \& Beers 1993). 


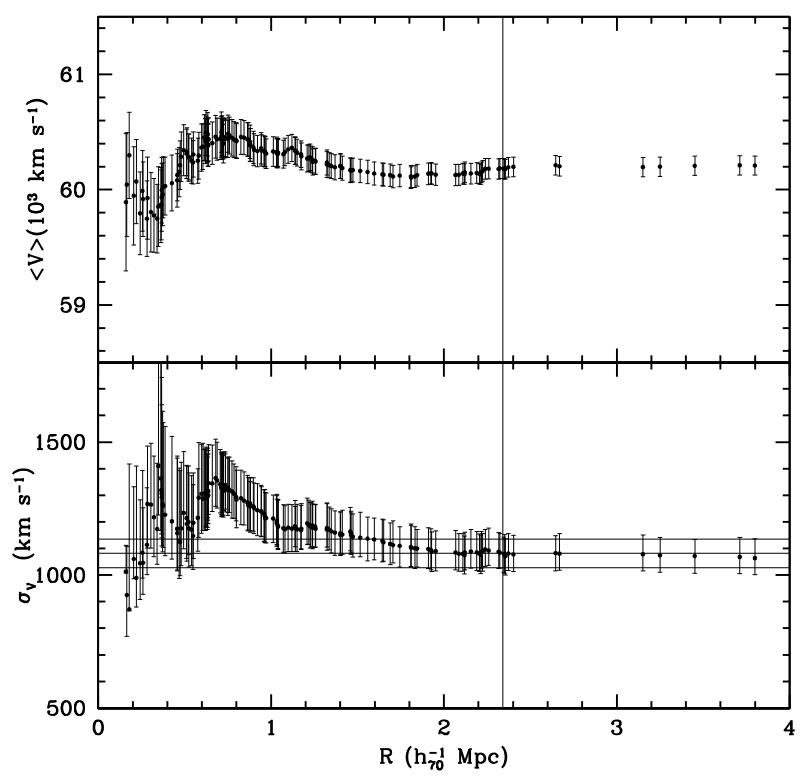

Fig. 6. Integral profiles of mean velocity (upper panel) and LOS velocity dispersion (lower panel). The mean and dispersion at a given (projected) radius from the cluster center is estimated by considering all galaxies within that radius (the first point is obtained on the basis of the five galaxies close to the cluster center). The error bands at the $68 \% \mathrm{c} .1$. are shown. In the lower panel, the horizontal line represents the X-ray temperature with the respective 90 per cent errors (Govoni et al. 2004) transformed in $\sigma_{\mathrm{v}}$ assuming the density-energy equipartition between gas and galaxies, i.e. $\beta_{\text {spec }}=1$ (see text).

Table 2. Results of the weighted gap analysis for the whole sample and for the MS subsystem.

\begin{tabular}{lcccc}
\hline \hline Sample & $N_{\text {gals pre }}, N_{\text {gals aft }}$ & $\begin{array}{c}v_{\text {pre }}, v_{\text {aft }} \\
\mathrm{km} \mathrm{s}^{-1}\end{array}$ & Size & Prob. \\
\hline Whole Sample & 145,22 & 61547,61964 & 3.81 & $5.0 \mathrm{E}-4$ \\
MS & 30,30 & 59059,59163 & 2.52 & $1.4 \mathrm{E}-2$ \\
MS & 30,33 & 59722,59783 & 2.35 & $3.0 \mathrm{E}-2$ \\
MS & 33,52 & 60440,61548 & 2.34 & $3.0 \mathrm{E}-2$ \\
\hline
\end{tabular}

Then we investigate the presence of gaps in the velocity distribution. A weighted gap in the space of the ordered velocities is defined as the difference between two contiguous velocities, weighted by the location of these velocities with respect to the middle of the data. We obtain values for these gaps relative to their average size, precisely the midmean of the weighted-gap distribution. We look for normalized gaps larger than 2.25 since in random draws of a Gaussian distribution they arise at most in about $3 \%$ of the cases, independent of the sample size (Wainer and Schacht 1978; see also Beers et al. 1991). We detect a significant gap (at the $99.95 \%$ c.1.) which separates the main cluster from a group of 22 high velocity galaxies (see Fig. 5 and the first line of Table 2). For each gap Table 2 lists the number of galaxies for the group before the gap and that after the gap (Col. 2); the velocity boundaries before and after the gap (Col. 3); the size of the gap (Col. 4); the probability of finding such a gap in a Gaussian distribution (Col. 5). Hereafter we define MS the main system with the 145 galaxies having low velocities and HGV the group with the 22 galaxies having high velocities (see Table 3 for their main kinematical properties).

As for the spatial distribution, there is no difference between the galaxies of the HVG and the MS (according to the 2D Kolmogorov-Smirnov test - hereafter 2DKS - test -
Table 3. Global properties of the whole sample, the MS and the HVG.

\begin{tabular}{|c|c|c|c|c|c|}
\hline Sample & & $\begin{array}{l}\langle v\rangle \\
\mathrm{km} \mathrm{s}^{-1}\end{array}$ & $\begin{array}{l}\sigma_{\mathrm{v}} \\
\mathrm{km} \mathrm{s}^{-1}\end{array}$ & $\begin{array}{c}R_{\mathrm{vir}} \\
h_{70}^{-1} \mathrm{Mpc} \\
\end{array}$ & $\begin{array}{l}\text { Mass }\left(<R_{\text {vir }}\right) \\
h_{70}^{-1} 10^{14} M_{\odot}\end{array}$ \\
\hline Whole system & 167 & $60209 \pm 82$ & $1066_{-61}^{+67}$ & 2.34 & $17 \pm 2$ \\
\hline MS & 145 & $59978 \pm 67$ & $812_{-46}^{+35}$ & 1.79 & $8 \pm 2$ \\
\hline HVG & 22 & $62419 \pm 74$ & $338_{-84}^{+225}$ & 0.74 & $0.6_{-0.3}^{+0.8}$ \\
\hline
\end{tabular}

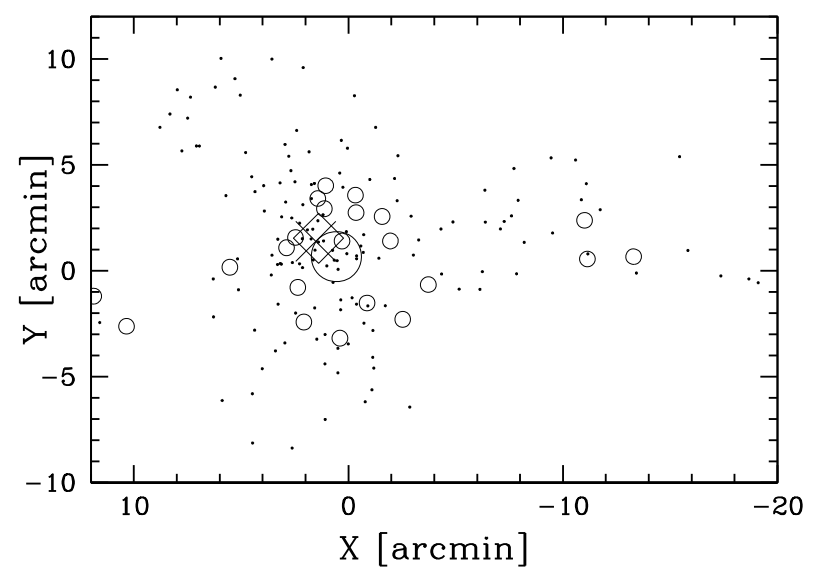

Fig. 7. Spatial distribution on the sky of the 167 galaxies of the whole cluster showing the two groups recovered by the weighted gap analysis. Dots and small circles indicate the main system (MS) and the high velocity group (HVG) galaxies. Large cross, rotated square and circle indicate the optical centers of the whole cluster, the MS and the HVG, respectively. The X-ray peak is taken as the cluster center.

Fasano \& Franceschini 1987, see Fig. 7). However, when considering the clustercentric distances, they differ at the $97.5 \%$ c.l. according to the 1D Kolmogorov-Smirnov test (hereafter 1DKStest, see e.g. Press et al. 1992) with the HVG galaxies being, on average, closer to the cluster (X-ray) center. Accordingly, while the optical (biweight) center of the MS lies very close to the optical center of the whole cluster, the optical (biweight) center of the HVG is closer to the X-ray cluster center (see Fig. 7).

We also use the results of the gap analysis to determine the first guess when using the Kaye's mixture model (KMM) test to find a possible group partition of the velocity distribution (as implemented by Ashman et al. 1994). The KMM algorithm fits a user-specified number of Gaussian distributions to a dataset and assesses the improvement of that fit over a single Gaussian. In addition, it provides the maximum-likelihood estimate of the unknown n-mode Gaussians and an assignment of objects into groups. We find a two-groups partition which is a significantly better descriptor of the velocity distribution with respect to a single Gaussian at the $95 \%$ cl.. The cluster partition is similar to that indicated by the above weighted gap analysis detecting two groups with 146 and 21 galaxies.

\subsubsection{Dressler-Shectman statistics}

We also analyze substructure combining galaxy velocity and position information. We compute the $\Delta$-statistics devised by Dressler \& Shectman (1988, hereafter DS). We find a significant indication of DS substructure (at the 97\% c.l. using 1000 Monte Carlo simulations; see e.g. Boschin et al. 2004). Figure 8 shows the distribution on the sky of all galaxies, each marked by a circle: the larger the circle, the larger the deviation $\delta_{i}$ of the 


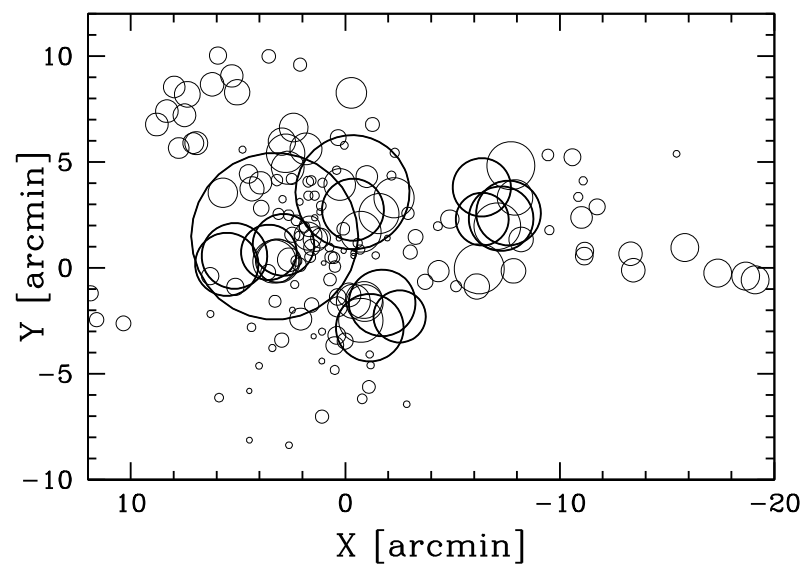

Fig. 8. Spatial distribution of the 167 cluster members, each marked by a circle: the larger the circle, the larger is the deviation $\delta_{i}$ of the local parameters from the global cluster parameters, i.e. there is more evidence for substructure (according to the Dressler \& Shectman test, see text). Heavy circles indicate those with $\delta_{i} \geq 2.5$.

local kinematical parameters from the global cluster parameters, i.e. the higher the evidence for substructure.

To better point out galaxies belonging to substructures, we resort to the technique developed by Biviano et al. (2002, see also Boschin et al. 2006; Girardi et al. 2006), who used the individual $\delta_{i}$-values of the DS method. The critical point is to determine the value of $\delta_{i}$ that optimally indicates galaxies belonging to substructure. To this aim we consider the $\delta_{i}$-values of all 1000 Monte Carlo simulations used above. The resulting distribution of $\delta_{i}$ is compared to the observed one finding a difference at the $99 \%$ c.l. according to the 1DKS-test. The "simulated" distribution is normalized to produce the observed number of galaxies and compared to the observed distribution in Fig. 9: the latter shows a tail at high values. Selecting galaxies with $\delta_{i} \leq 2.5$ the 1DKS-test gives only a marginal difference between real and simulated galaxies (at the $93 \%$ c.l.) suggesting that galaxies with $\delta_{i}>2.5$ presumably are in substructures. These galaxies are indicated with heavy circles in Fig. 8 showing four subclumps (at northern, eastern, southern and distant western cluster regions).

\subsubsection{Analysis of velocity dispersion profiles}

Finally we analyze the kinematical properties of galaxy populations located in different spatial regions of the cluster. We compute the profiles of mean velocity and velocity dispersion of galaxy systems surrounding the lensing mass peaks listed by M07 (see Fig. 10). This allows an independent analysis of the possible individual galaxy clumps. A quasi flat profile is expected in the case of a relaxed system with isotropic orbits for galaxies (e.g. Girardi et al. 1998). Although an increasing/decreasing profile might be due to particular orbits of galaxies in a relaxed system (e.g. Girardi et al. 1998; Biviano \& Katgert 2004), here this is likely connected with the presence of substructure. As for an increasing profile, this might be simply induced by the contamination of the galaxies of a close, secondary clump having a different mean velocity (e.g. Girardi et al. 1996; Girardi et al. 2006). This hypothesis can be investigated by looking at the behavior of the mean velocity profile. In fact, if the $\sigma_{\mathrm{v}}$ profile increases due to the contamination of a close clump, for the same reason and at about the same radius, the $\langle v\rangle$ profile should increase/decrease. As for a decreasing profile, this might be likely due to the projection effect of a few clumps

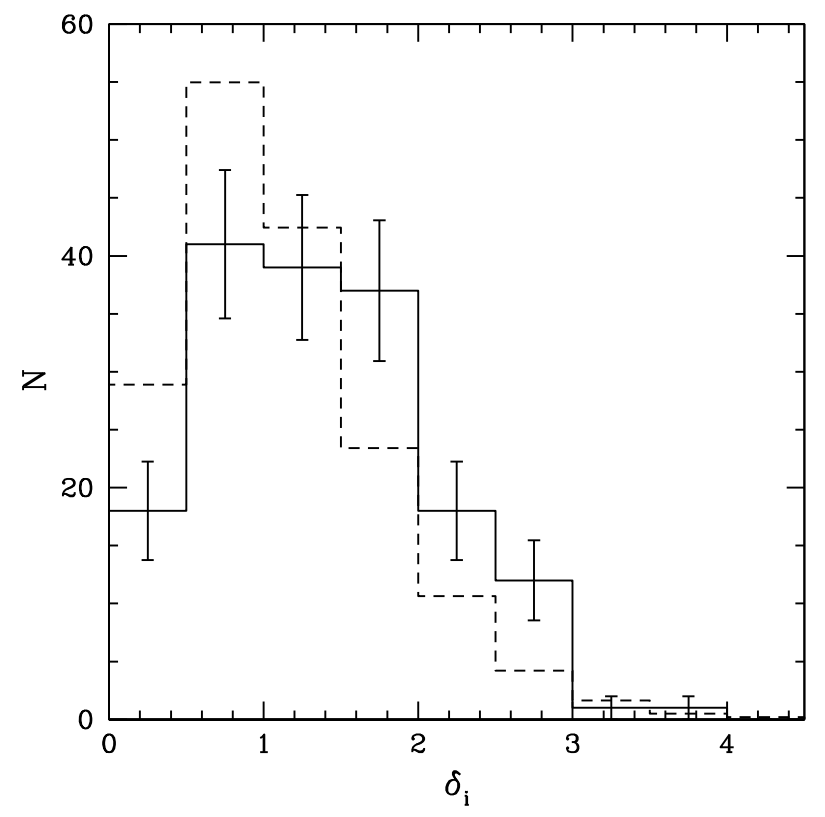

Fig. 9. The distribution of $\delta_{i}$ deviations of the Dressler-Shectman analysis for the 167 member galaxies. The solid line represents the observations, the dashed line the distribution for the galaxies of simulated clusters, normalized to the observed number.

centered around the center of the system and having different mean velocities, i.e. somewhat aligned with the LOS or, alternatively, of a large scale structure (LLS) elongated along the LOS (e.g. a LLS filament).

The inspection of Fig. 10 shows that the $\sigma_{\mathrm{v}}$ profiles of peaks Nos. 1, 2 and 5 sharply increase with the distance from the peak position. Simultaneously, the $\langle\mathrm{v}\rangle$ profiles decline (peak No. 1) or increase (peaks Nos. 2 and 5). For each of these clumps we attempt to detect the region likely not contaminated by other clumps - and thus reliable for kinematical analysis - as the region before the sharp increasing of the $\sigma_{\mathrm{v}}$ profile (see the arrows in Fig. 10, for peaks Nos. 1, 2, and 5). No conclusion can be driven for peaks Nos. 3 and 4 where the $\sigma_{\mathrm{v}}$ profile is decreasing.

\subsection{Substructure of the main system}

Here we present the results of our substructure analyses applied to the 145 galaxies of the main system (MS), i.e. rejecting the galaxies of the high velocity group (HVG) which might mask the real cluster structure.

Table 4 and Fig. 11 summarize kinematical and spatial properties of the subclumps we detect in the MS. In particular, Table 4 lists for each clump the number of galaxies (Col. 2); the mean velocity and its jacknife error (Col. 3); the velocity dispersion and its bootstrap error (Col. 4); the luminous galaxies contained within the analyzed clump (Col. 5); the name of the corresponding structure discussed in Sect. 5 (Col. 6). The following subsections show the results recovered for each of the three methods of analysis.

\subsubsection{Velocity distribution}

The velocity distribution of the MS is negatively skewed (at the c.l. of 90-95\%, skewness $=-0.330$ ) and light-tailed (at the c.l. of $90-95 \%$, kurtosis $=2.347$ ). The W-test (Shapiro \& Wilk 1965) rejects the null hypothesis of a Gaussian parent distribution at the $>99.9 \%$ c.l. 


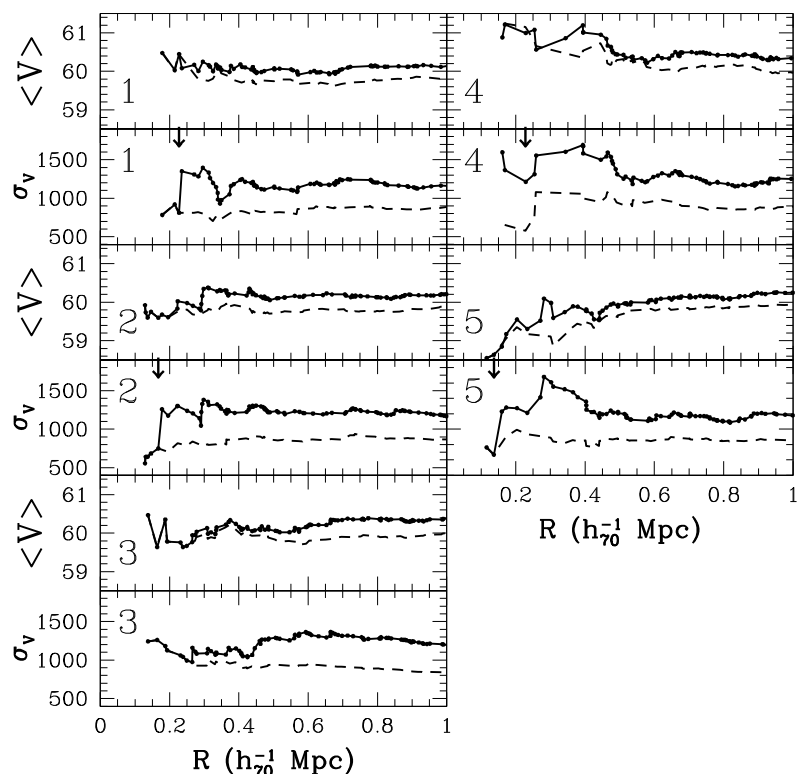

Fig. 10. Kinematical profiles of the galaxy clump surrounding the lensing mass peaks Nos. 1-5 listed by M07. For each peak integral mean velocity (in units of $10^{3} \mathrm{~km} \mathrm{~s}^{-1}$ ) and LOS velocity dispersion (in units of $\mathrm{km} \mathrm{s}^{-1}$ ) profiles are shown in upper and lower panels, respectively. The technique is the same adopted in Fig. 6, but we omit errors for the sake of clarity. The solid and dashed lines join the values obtained using all cluster galaxies and only galaxies belonging to the MS, respectively. The arrows indicate the regions likely not contaminated from other subclumps (see Sects. 4.3.3 and 4.4.3)

Table 4. Results of the substructure analysis of the MS.

\begin{tabular}{|c|c|c|c|c|c|}
\hline Gal.Clump & $N_{g}$ & $\begin{array}{l}\langle v\rangle \\
\mathrm{km} \mathrm{s}^{-1}\end{array}$ & $\begin{array}{l}\sigma_{\mathrm{v}}^{a} \\
\mathrm{~km} \mathrm{~s}^{-1}\end{array}$ & $\begin{array}{l}\text { Lum.gals } \\
\text { ID }\end{array}$ & Structures \\
\hline $\mathrm{V} 1^{b}$ & 30 & $58568 \pm 56$ & $300_{-46}^{+54}$ & 205 & $\mathcal{E}$ \\
\hline $\mathrm{V} 2^{b}$ & 30 & $59455 \pm 18$ & $95_{-6}^{+6}$ & 44,170 & $\mathcal{N E} 2+\mathcal{W}$ \\
\hline $\mathrm{V} 3^{b}$ & 33 & $60113 \pm 19$ & $106_{-13}^{+13}$ & $204,160,137$ & $\mathcal{N E} 1$ \\
\hline $\mathrm{V} 4^{b}$ & 52 & $60920 \pm 33$ & $238_{-36}^{+7}$ & 106,264 & $\mathcal{S W}+C$ \\
\hline DS-N & 4 & $58427 \pm 272$ & $415_{-415}^{+478}$ & - & $\mathcal{N}$ \\
\hline DS-S & 4 & $61165 \pm 519$ & $791_{-471}^{+323}$ & 106 & $S W$ \\
\hline DS-E & 7 & $58686 \pm 292$ & $682_{-239}^{+356}$ & 205 & $\mathcal{E}$ \\
\hline DS-W & 4 & $59635 \pm 249$ & $380_{-112}^{+83}$ & - & $W$ \\
\hline P 1 & 7 & $60448 \pm 349$ & $811_{-71}^{+278}$ & 204 & $\mathcal{N E} 1$ \\
\hline P 2 & 9 & $59596 \pm 276$ & $749_{-88}^{+186}$ & 170,160 & $\mathcal{N E} 2$ \\
\hline P 4 & 6 & $61447 \pm 283$ & $579_{-151}^{+523}$ & 106 & $S W$ \\
\hline P 5 & 6 & $58634 \pm 325$ & $668_{-187}^{+570}$ & 205 & $\mathcal{E}$ \\
\hline
\end{tabular}

${ }^{a}$ We use the biweight and the gapper estimators by Beers et al. (1990) for samples with $N_{g} \geq 15$ and with $N_{g}<15$ galaxies, respectively (see also Girardi et al. 1993); ${ }^{b}$ the estimate of $\sigma_{\mathrm{V}}$ should be considered a lower limit in these samples (see text).

We detect three marginally significant gaps which divide the MS in four groups of 30, 30, 33 and 52 galaxies (see Fig. 5 and Table 2), hereafter defined as V1, V2, V3 and V4 from low to high velocities. When compared two by two through the 2DKS-test, these groups differ in spatial distribution: the $\mathrm{V} 1$ group differs both from the V2 and V3 groups (at the $98 \%$ and $94 \%$ c.l., respectively); the V4 group differs from the V3 group (at the $94 \%$ c.l.). For each of the these groups Fig. 12 shows the spatial distribution of galaxies and the corresponding peak according to the 2D DEDICA procedure. For the V2 group,

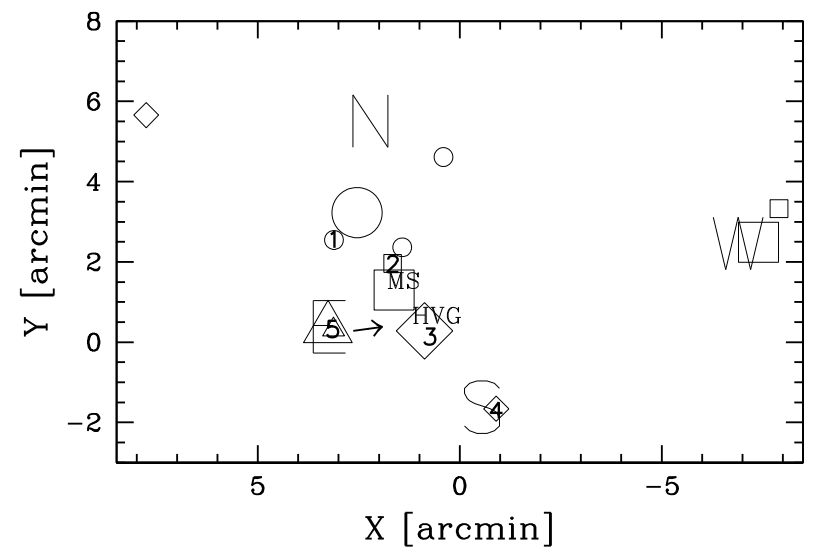

Fig. 11. Summary of spatial distribution of cluster substructure. Labels "MS" and "HVG" indicate the optical centers of the MS and of the HVG, respectively. Other symbols refer to galaxy subclumps found in the MS using several, different approaches. Triangles, squares, circles and rotated squares indicate 2D DEDICA peaks (large symbols) and luminous galaxies (small symbols) of the V1, V2, V3 and V4 clumps. Labels "N", "S", "E" and "W" indicate the (biweight) centers of the four DS clumps. Labels " 1 ", “" 2 ", "3", " 4 " and " 5 " indicate the corresponding lensing mass peaks listed by M07: around peaks Nos. 1, 2, 4 and 5 we detect the P1, P2, P4 and P5 clumps. On the base of the location of these subclumps and their velocities in Table 4 we discuss the presence of five main structures $(\mathcal{N E} 1, \mathcal{N} \mathcal{E} 2, \mathcal{S W}, \mathcal{E}$ and $\mathcal{W}$ centered around the labels "1", "2", "4", "5" and "W", respectively) and of two minor structures ( $\mathcal{N}$ and $C$ roughly located around the label " $\mathrm{N}$ " and the large rotated square, respectively), see Sect. 5. The arrow indicates the head tail radiogalaxy ID 184 with the direction of its tail. The plot is centered on the X-ray cluster center.

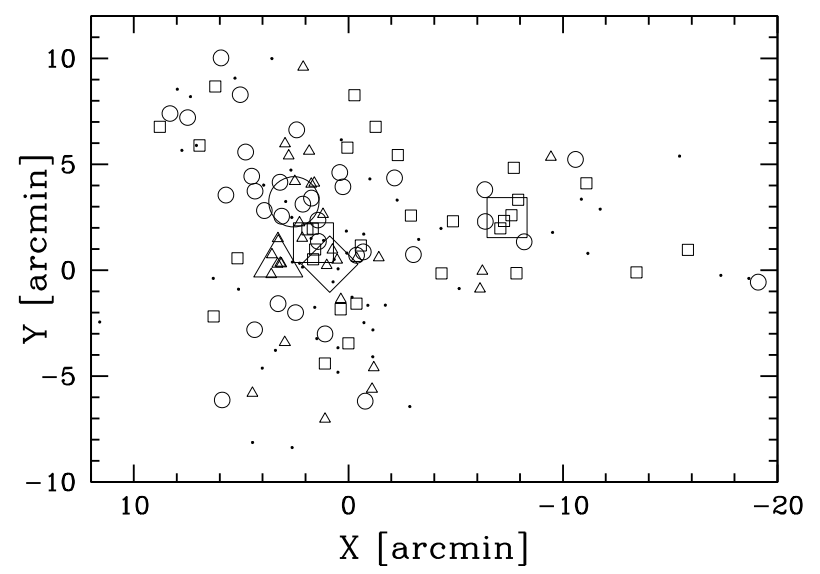

Fig. 12. Spatial distribution on the sky of the 145 galaxies belonging to the MS showing the four groups recovered by the weighted gap analysis. Small triangles, squares, circles and dots indicate galaxies of the V1, V2, V3 and V4 groups, respectively. Large triangle, (2) squares, circle and rotated square indicate DEDICA peaks of the V1, V2, V3 and V4 galaxy distributions.

we find and plot two peaks of comparable significance. Figure 11 shows the position of these peaks in relation to the lensing mass peaks listed by M07. Each of these groups contains one or more luminous galaxies and, with the exception of V4, each group hosts one correspondent luminous galaxy close to the respective peak in the galaxy distribution (see Fig. 11). Properties of groups recovered by kinematical analysis are listed in Table 4. For the V1, V2, V3 and V4 groups, the membership assignment might lead to an artificial truncation of the tails of the 


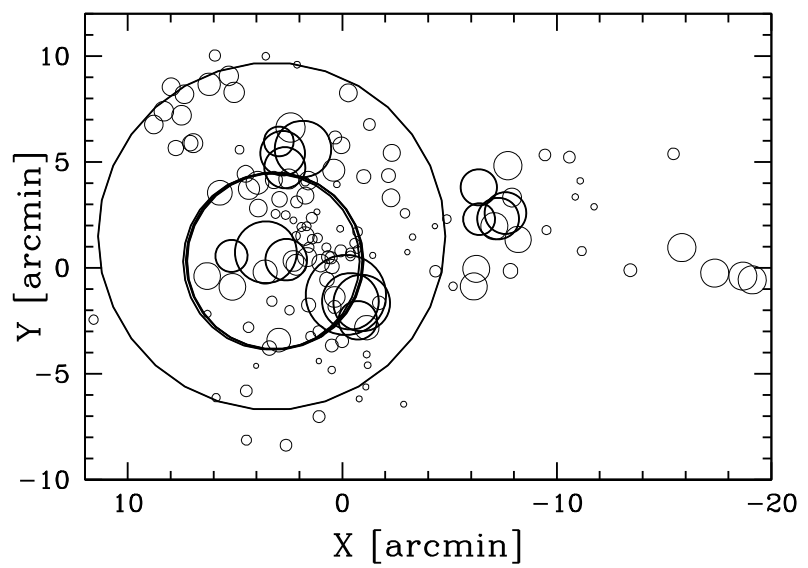

Fig. 13. The result of Dressler-Shectman analysis as in Fig. 8, but for the 145 members of the MS. Here heavy circles indicate galaxies with $\delta_{i}>1.95$ used to define the DS-N, DS-S, DS-E and DS-W clumps (see text).

distributions; thus the values of velocity dispersion should be considered lower limits (e.g. Bird 1994).

Using the results of the gap analysis to determine the first guess of the KMM algorithm we find that a four-group partition is a much better descriptor of the velocity distribution with respect to a single Gaussian at the $99 \% \mathrm{cl}$.. In particular, the cluster partition is similar to that indicated by the above weighted-gap analysis separating the MS in groups of 33, 27, 35 and 50 galaxies.

\subsubsection{Dressler-Shectman statistics}

The DS test on the MS gives a very marginal indication of substructure (at the $91 \%$ c.l.). However, as for the location of substructures, the DS plot of the MS is similar to that recovered for the whole sample (cf. Figs. 8 and 13). The main difference is the disappearance of the northern substructure very close to the X-ray cluster center which was likely due to the galaxies of the HVG. The other three subclumps are still present (at eastern, southern and distant western cluster regions). Moreover, the analysis of the MS shows a northern subclump 5' from the X-ray cluster center. In order to better investigate the properties of DS substructure we select the galaxies with the highest $\delta_{i}$ in such a way that at least four galaxies can be assigned in a unique way to each of the four DS subclumps. This leads to 19 galaxies with $\delta_{i}>1.95$. The kinematical properties of the four DS subclumps are listed in Table 4. Figure 11 shows the position of their (biweight) centers.

\subsubsection{Analysis of velocity dispersion profiles}

We also reanalyze the kinematical properties of galaxies surrounding the lensing mass peaks listed by M07 (see Fig. $10-$ dashed lines). For all the peaks where we have defined a region likely uncontaminated by other clumps in Sect. 4.3 .3 (peaks Nos. 1, 2 and 5), we confirm that these regions are devoid of HVG galaxies. Moreover, now we also find a sharp increase of the $\sigma_{\mathrm{v}}$ profile for peak No. 4 and we define a likely non contaminated region for the subsystem corresponding to this peak, too. The groups formed by galaxies within the uncontaminated fiducial regions are referred to as P1, P2, P4 and P5 and their kinematical properties are shown in Table 4.
In the case of peak No. 3 we still find a decreasing $\sigma_{\mathrm{v}}$ profile; thus we find no evidence for an individual, dynamically important structure around this peak.

\section{Cluster structure and dynamics}

The value we find for the global velocity dispersion of the cluster members, $\sigma_{\mathrm{v}}=1066_{-61}^{+67}$, is in agreement with previous analyses (Carlberg et al. 1996; Borgani et al. 1999; Proust et al. 2000; Mezzetti \& Girardi 2001). This value of the velocity dispersion is also comparable to the average X-ray temperature assuming the density-energy equipartition between gas and galaxies, i.e. $\beta_{\text {spec }}=1^{3}$, see Fig. 6 - lower panel.

We analyze the cluster structure using the velocity distribution analysis (weighted gap technique and KMM method), the Dressler-Shectman statistics and the analysis of the velocity dispersion profiles. The structure of A520 is definitely very complex and thus likely far from the dynamical equilibrium. The agreement between $\sigma_{\mathrm{v}}$ and $T_{\mathrm{X}}$ might be due to an enhancement of both quantities and, as already pointed out by M07, gross properties and scaling relations are not always useful indicators of the dynamical state of clusters.

Hereafter we analyze and discuss the structure of A520 starting from the simplest hypothesis and then adding some degrees of complexity.

\subsection{Mass estimate}

Making the usual assumptions (cluster sphericity, dynamical equilibrium, that the galaxy distribution traces the mass distribution), one can compute virial global quantities. Following the prescriptions of Girardi \& Mezzetti (2001), we assume for the radius of the quasi-virialized region $R_{\mathrm{vir}}=0.17 \times \sigma_{\mathrm{v}} / H(z)=$ $2.34 h_{70}^{-1} \mathrm{Mpc}-$ see their Eq. (1) after introducing the scaling with $H(z)$ (see also Eq. (8) of Carlberg et al. 1997, for $R_{200}$ ). Thus the cluster is sampled out - although in a non-homogeneous way - to $R_{200}$. We compute the virial mass (Limber \& Mathews 1960; see also, e.g., Girardi et al. 1998):

$M=3 \pi / 2 \cdot \sigma_{\mathrm{v}}^{2} R_{\mathrm{PV}} / G-\mathrm{SPT}$,

where SPT is the surface pressure term correction (The \& White 1986 ), and $R_{\mathrm{PV}}$ is a projected radius (equal to two times the projected harmonic radius).

The estimate of $\sigma_{\mathrm{v}}$ is robust when computed within a large cluster region (see Fig. 6). The value of $R_{\mathrm{PV}}$ depends on the size of the sampled region and possibly on the quality of the spatial sampling (e.g., whether the cluster is uniformly sampled or not). Considering the 155 galaxies within $R_{\text {vir }}$ we obtain $R_{\mathrm{PV}}=$ $(1.72 \pm 0.09) h_{70}^{-1} \mathrm{Mpc}$, where the error is obtained via the jacknife procedure. The value of SPT strongly depends on the radial component of the velocity dispersion at the radius of the sampled region and could be obtained by analyzing the (differential) velocity dispersion profile, although this procedure would require several hundred galaxies. We decide to assume a 20\% SPT correction as obtained in the literature by combining data on many clusters sampled out to about $R_{\text {vir }}$ (Carlberg et al. 1997; Girardi et al. 1998). We compute $M\left(<R_{\text {vir }}=2.34 h_{70}^{-1} \mathrm{Mpc}\right)=(1.7 \pm$ $0.2) \times 10^{15} h_{70}^{-1} M_{\odot}$.

Since the cluster center of A520 is not well defined and the spatial sampling is not complete and homogeneous within $R_{\text {vir }}$,

\footnotetext{
${ }^{3} \beta_{\text {spec }}=\sigma_{\mathrm{v}}^{2} /\left(k T / \mu \mathrm{m}_{\mathrm{p}}\right)$ with $\mu=0.58$ the mean molecular weight and $m_{\mathrm{p}}$ the proton mass.
} 
one could use an alternative estimate of $R_{\mathrm{PV}}$ on the basis of the knowledge of the galaxy distribution. Following Girardi et al. (1998; see also Girardi \& Mezzetti 2001) we assume a King-like distribution with parameters typical of nearby/medium-redshift clusters: a core radius $R_{\mathrm{c}}=1 / 20 \times R_{\mathrm{vir}}$ and a slope-parameter $\beta_{\text {fit }}=0.8$, i.e. the volume galaxy density at large radii goes as $r^{-3 \beta_{\mathrm{fit}}}=r^{-2.4}$. We obtain $R_{\mathrm{PV}}=1.74 h_{70}^{-1} \mathrm{Mpc}$, where a $25 \%$ error is expected (Girardi et al. 1998). The mass recovered by this method is then $M\left(<R_{\text {vir }}=2.34 h_{70}^{-1} \mathrm{Mpc}\right)=$ $(1.7 \pm 0.5) \times 10^{15} h_{70}^{-1} M_{\odot}$ in excellent agreement with the above direct estimate.

Our analysis of the cluster velocity distribution detects the presence of a high velocity group (HGV) with a relative restframe LOS velocity of $v_{\mathrm{rf}} \sim 2000 \mathrm{~km} \mathrm{~s}^{-1}$ with respect to the main system (MS), see Sect. 4.3.1. Therefore we might think that the cluster is better described by the combination of the MS and the HVG, considered as two separated entities. Assuming the dynamical equilibrium for both the MS and the HVG we can compute independent virial radii and masses $M\left(<R_{\text {vir }}=1.79 h_{70}^{-1} \mathrm{Mpc}\right)=(8 \pm 2) \times 10^{14} h_{70}^{-1} M_{\odot}$ and $M\left(<R_{\mathrm{vir}}=0.74 h_{70}^{-1} \mathrm{Mpc}\right)=\left(0.6_{-0.3}^{+0.8}\right) \times 10^{14} h_{70}^{-1} M_{\odot}$, respectively.

To compare the mass estimates derived for the two above cluster models (relaxed cluster and the MS+HVG system) we consider the mass values within $1 h_{70}^{-1} \mathrm{Mpc}$. To rescale our mass estimates we assume that the system is described by a King-like mass distribution (see above) or, alternatively, a NFW profile where the mass-dependent concentration parameter $c$ is taken from Navarro et al. (1997) and rescaled by the factor $1+z$ (Bullock et al. 2001; Dolag et al. 2004), i.e. $c=4.16$, 4.96 and 6.18 for the whole cluster, the MS and the HVG, respectively. The relaxed cluster model leads to a mass of $M\left(<1 h_{70}^{-1} \mathrm{Mpc}\right)=$ (7.2-9.6) $\times 10^{14} h_{70}^{-1} M_{\odot}$ while the addition of the MS and the HVG masses leads to a mass of $M\left(<1 h_{70}^{-1} \mathrm{Mpc}\right)=(4.0-6.8)$ $\times 10^{14} h_{70}^{-1} M_{\odot}$ [where the mass range includes a $1 \sigma$ error on the original $M\left(<R_{\text {vir }}\right)$ estimate].

Using the above rescaling we can also compare our results with the estimates recovered from X-ray and gravitational lensing analyses. Lewis et al. (1999) used the ROSAT $\mathrm{X}$-ray surface brightness and ASCA temperature to estimate a mass of $M\left(<1.764 \quad h_{70}^{-1} \mathrm{Mpc}\right)=(11.3 \pm 1.1)$ $\times 10^{14} \quad h_{70}^{-1} M_{\odot} \quad$ (the ROSAT-PSPC estimate is converted in our cosmology). X-ray mass is intermediate between our estimates since we obtain $M\left(<1.764 \quad h_{70}^{-1} \mathrm{Mpc}\right)=(12-$ 15) $\times 10^{14} \quad h_{70}^{-1} M_{\odot}$ and $(6.1-10) \times 10^{14} h_{70}^{-1} M_{\odot}$ for the two cluster models. As for gravitational lensing, Dahle et al. (2002) obtained the projected mass $M_{\text {proj }}\left(<1.111 h_{70}^{-1} \mathrm{Mpc}\right)=$ $11.7_{-2.3}^{+3.9} \times 10^{14} h_{70}^{-1} M_{\odot} \quad$ (see their Fig. 50 with conversion in our cosmology). Our projected mass estimates for the two cluster models are $M_{\text {proj }}\left(<1.111 h_{70}^{-1} \mathrm{Mpc}\right)=(12-18)$ $\times 10^{14} h_{70}^{-1} M_{\odot}$ and (5.4-11) $\times 10^{14} h_{70}^{-1} M_{\odot}$, where to make the projection we have considered that the cluster mass distribution is truncated at one or at two virial radii.

\subsection{Main system and high velocity group: relative dynamics}

Continuing with the assumption of a cluster formed by the MS and the HVG, we investigate their relative dynamics. We use different analytic approaches which are based on an energy integral formalism in the framework of locally flat spacetime and Newtonian gravity (e.g. Beers et al. 1982). The values of the relevant observable quantities for the two-clumps system are: the relative LOS velocity in the rest frame, $V_{\mathrm{rf}}=2033 \mathrm{~km} \mathrm{~s}^{-1}$ (as recovered from the MS and the HVG); the projected linear distance between the two clumps, $D=0.21 h_{70}^{-1} \mathrm{Mpc}$ (as recovered from optical centers of the MS and the HVG); the mass of the system obtained by adding the masses of the two subclusters each within its virial radius, $\log M_{\text {sys }}=14.9154_{-0.1512}^{+0.1264}$ (see Table 3).

First, we consider the Newtonian criterion for gravitational binding stated in terms of the observables as $V_{\mathrm{r}}^{2} D \leq$ $2 G M_{\text {sys }} \sin ^{2} \alpha \cos \alpha$, where $\alpha$ is the projection angle between the plane of the sky and the line connecting the centers of the two clumps. The thin curve in Fig. 14 separates the bound and unbound regions according to the Newtonian criterion (above and below the curve, respectively). Considering the value of $M_{\text {sys }}$, the MS+HVG system is bound between $21^{\circ}$ and $83^{\circ}$; the corresponding probability, computed considering the solid angles (i.e., $\int_{21}^{83} \cos \alpha \mathrm{d} \alpha$ ), is $63 \%$.

Then, we apply the analytical two-body model introduced by Beers et al. (1982) and Thompson (1982; see also Lubin et al. 1998, for a recent application). This model assumes radial orbits for the clumps with no shear or net rotation of the system. Furthermore, the clumps are assumed to start their evolution at time $t_{0}=0$ with separation $d_{0}=0$, and are moving apart or coming together for the first time in their history; i.e. we are assuming that we are seeing the HVG prior to merging with the MS (at the time $t=11.022$ Gyr at the cluster redshift, see Wright 2006). The bimodal model solution gives the total system mass $M_{\text {sys }}$ as a function of $\alpha$ (e.g. Gregory \& Thompson 1984). Figure 14 compares the bimodal-model solutions with the observed mass of the system. The present bound outgoing solutions (i.e. expanding), BO, are clearly inconsistent with the observed mass. The possible solutions span these cases: the bound and present incoming solution (i.e. collapsing), $\mathrm{BI}_{\mathrm{a}}$ and $\mathrm{BI}_{\mathrm{b}}$, and the unbound-outgoing solution, UO. For the incoming case there are two solutions because of the ambiguity in the projection angle $\alpha$. We compute the probabilities associated to each solution assuming that the region of $M_{\text {sys }}$ values between the uncertainties are equally probable for individual solutions: $P_{\mathrm{BIa}} \sim 88 \%$, $P_{\mathrm{BIb}} \sim 12 \%, P_{\mathrm{UO}} \sim 7 \times 10^{-6} \%$. Thus it is very likely that the HVG lies in front of the cluster just infalling onto it.

Notice, however, that the centers of the HVG and the MS are not well determined. The HVG has too small a number of galaxies for a precise center determination. As for the MS center, we might adopt the X-ray cluster center instead of the optical cluster center. These uncertainties do not change the bulk of our results. For instance, in the case where we assume that the X-ray center is the MS center, i.e. a smaller projected linear distance between the two clumps $\left(D=0.1 h_{70}^{-1} \mathrm{Mpc}\right)$, the effect is to increase the boundary probability (at $75 \%$ c.l. for the Newtonian model) and to yield more extreme values for the bound solutions ( $\alpha \sim 15$ and 85 degrees). Also, possible underestimates of the masses (e.g., if the MS and the HVG actually extend outside of the virial radii we estimate for them) would lead to binding probabilities larger than those computed above, as well as more extreme values for $\alpha$. Thus the analysis here displayed should be considered a lower limit for our conclusions in Sect. 5.5, where we propose the existence of a cluster accretion along the LOS (i.e. $\alpha$ close to 90 degrees).

\subsection{NE-SW merger}

Although the presence of the HVG system is maybe the most important for the optical virial mass computation, we find that A520 shows a much more complex structure. In fact, we detect 


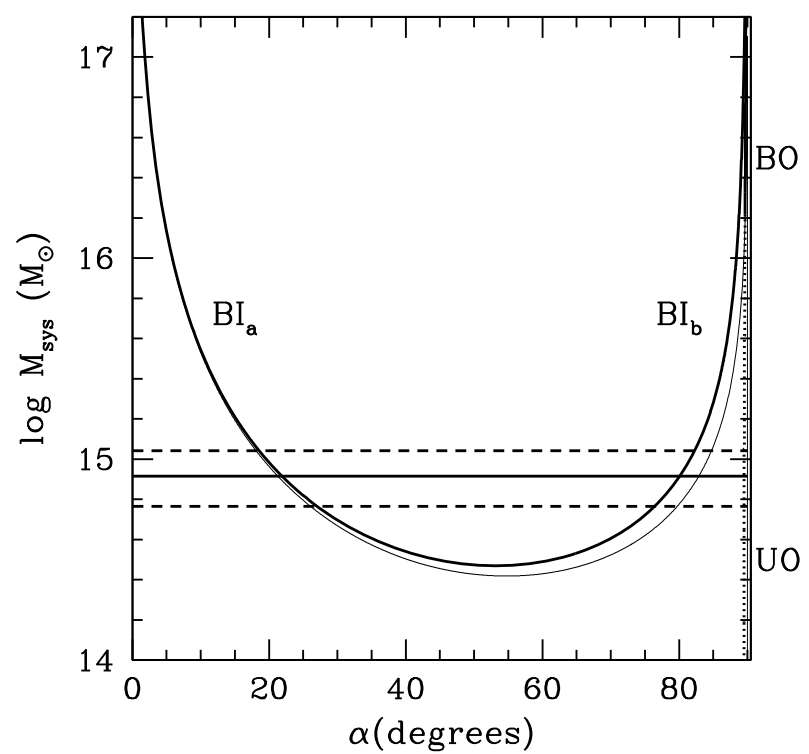

Fig. 14. System mass vs. projection angle for bound and unbound solutions (solid and dotted curves, respectively) of the two-body model applied to the MS and the HVG subsystems. Labels $\mathrm{BI}_{\mathrm{a}}$ and $\mathrm{BI}_{\mathrm{b}}$ indicate the bound and incoming, i.e. collapsing solutions (the main part of the solid curve). Label BO indicates the bound outgoing, i.e. expanding solutions (the part of the solid curve which is roughly a vertical line). Label UO indicates the unbound outgoing solutions (the dotted curve which is roughly a vertical line). The horizontal lines give the observational values of the mass system and its uncertainties. The bound and unbound regions according to the Newtonian criterion are indicated, too (above and below the thin curve, respectively).

several subsystems in the MS along the NE-SW and E-W directions (see Figs. 1 and 11).

Clumps around lensing mass peaks Nos. 1, 2 and 4 of M07 define the direction of the likely merger along the NE-SW direction with the SW structure having crossed the NE structure (Markevitch et al. 2005).

Around peaks Nos. 1 and 2 we detect two structures (hereafter $\mathcal{N E} 1$ and $\mathcal{N E} 2$ ) using the analysis of the velocity distribution (V3 and V2) and velocity dispersion profiles (P1 and $\mathrm{P} 2$ ). These structures have a relative rest-frame LOS velocity of $\sim 700 \mathrm{~km} \mathrm{~s}^{-1}$. Each structure hosts, close to the center, a luminous galaxy having typical velocity of the structure (IDs 204 and 170 in $\mathcal{N} \mathcal{E} 1$ and $\mathcal{N} \mathcal{E} 2$, respectively).

Structures $\mathcal{N E} 1$ and $\mathcal{N E} 2$ are so close in space and in velocity that the "uncontaminated" regions detected by $\sigma_{\mathrm{v}}$ profiles are slightly superimposed (see Fig. 1). Moreover, at $\lesssim 0.5^{\prime}$ from ID 170 (the $\mathcal{N} \mathcal{E} 2$ central, luminous galaxy) we find the luminous galaxy ID 160 which belongs to V3 group, i.e. to the $\mathcal{N E} 1$ structure. Such close couples of galaxies having different velocities are often observed in clusters (Boschin et al. 2006; Barrena et al. 2007a) and are the likely tracers of a previous cluster merger. Indeed cluster merger is thought to be the cause of the formation of dumbbell galaxies (e.g. Beers et al. 1992; Flores et al. 2000). Therefore $\mathcal{N} \mathcal{E} 1+\mathcal{N} \mathcal{E} 2$ is likely to form a single, although not yet well relaxed, structure and represent the real, original main cluster. In fact, the combined velocity of $\mathcal{N} \mathcal{E} 1$ and $\mathcal{N} \mathcal{E} 2$ is $\sim 60000 \mathrm{~km} \mathrm{~s}^{-1}$, similar to the mean velocity of the MS, and the $\mathcal{N} 22$ position is close to the optical center of the MS. The dynamical importance of the $\mathcal{N} \mathcal{E} 1+\mathcal{N} \mathcal{E} 2$ structure explains why the SW structure has been reported to have suffered significant damage in the merging, as shown by the pieces of the cluster core detected in X-ray (Markevitch et al. 2005).
Around peak No. 4 we detect a structure (hereafter $\mathcal{S W}$ ) both using DS analysis (DS-S) and studying the velocity dispersion profile $(\mathrm{P} 4)$. It is characterized by a high velocity $v \sim$ $61300 \mathrm{~km} \mathrm{~s}^{-1}$, i.e. $v_{\mathrm{rf}} \sim+1100 \mathrm{~km} \mathrm{~s}^{-1}$ from the $\mathcal{N} \mathcal{E} 1+\mathcal{N} \mathcal{E} 2$ complex. $\mathcal{S W}$ is not individually detected in the velocity distribution. However, since it hosts close to its center one of the two luminous galaxies of the V4 group (ID 106 with $v=$ $61277 \mathrm{~km} \mathrm{~s}^{-1}$ ), SW is likely a part of V4 (see also discussion in Sect. 5.5).

A possible structure related to the NE-SW merger is DS-N (hereafter $\mathcal{N}$ ) having a small velocity $v \sim 58400 \mathrm{~km} \mathrm{~s}^{-1}$. It is only detected through the Dressler-Shectman analysis and does not contain a luminous galaxy; therefore we neglect it in the following discussion. Notice however that it roughly corresponds to the $N$ peak in the lensing mass map of Okabe \& Umetsu (2008).

To attempt a more detailed analysis of the NE-SW merger we apply the bimodal model considering the interaction between $\mathcal{N E} 1+\mathcal{N E} 2$ (likely corresponding to the main part of the MS, see above) and $\mathcal{S W}$ assuming we are looking at them after their core crossing as suggested from X-ray data. As parameters of the model we use a relative LOS velocity $V_{\mathrm{rf}}=1100 \mathrm{~km} \mathrm{~s}^{-1}$ and a relative projected distance $D=0.8 h_{70}^{-1} \mathrm{Mpc}$. We assume the MS mass as the mass for the whole system. In order to apply the two-body model we assume that the time $t_{0}=0$ with separation $d_{0}=0$ is the time of their core crossing and that we are seeing the cluster a few $10^{8}$ years after the merging. In fact, a few $10^{8}$ years is the time scale in which the relativistic electrons lose energy, i.e. the lifetime of the radio halo (e.g. Giovannini \& Feretti 2002). Figure 15 shows the results for a time of $t=0.2$ and $0.3 \mathrm{Gyr}$ after the core crossing. The likely bound solution is then an outgoing one, i.e. the SW structure is now behind the NE structure going away from it. In particular, an angle of $\alpha \sim 30$ leads to an outgoing velocity of $\sim 2200 \mathrm{~km} \mathrm{~s}^{-1}$.

The Mach number of the shock is $\mathcal{M}=v_{\mathrm{s}} / c_{\mathrm{s}}$, where $v_{\mathrm{s}}$ is the velocity of the shock and $c_{\mathrm{S}}$ in the sound speed in the pre-shock gas (see e.g. Sarazin 2002, for a review). In the stationary regime we can assume that $v_{\mathrm{s}}$ is the merger velocity $2200 \mathrm{~km} \mathrm{~s}^{-1}$. Assuming the equipartition of energy density between gas and galaxies and using the $\sigma_{\mathrm{v}}$ of the MS we obtain $c_{\mathrm{S}} \sim 812 \mathrm{~km} \mathrm{~s}^{-1}$ from the thermal velocity. Therefore we estimate $\mathcal{M} \sim 2.7$, which is in reasonable agreement with $\mathcal{M}=2.1_{-0.3}^{+0.4}$ and $\mathcal{M}=2.2_{-0.5}^{+0.9}$ recovered from X-ray data (Markevitch et al. 2005).

Notice that $t=0.2-0.3 \mathrm{Gyr}$ and $\alpha \sim 30$ degrees are larger, but comparable, to the merger parameters recovered by Markevitch et al. (2002), for a similar scenario in the cluster 1E0657-56 which hosts a radio halo and shows a bow shock, too (0.1-0.2 Gyr and 10-15 degrees). Indeed, this agreement is not casual since as noticed by Markevitch et al. (2005) to observe a shock front "one has to catch a merger at a very specific stage when the shock has not yet moved to the outer, low surface brightness regions and at a sufficiently small angle from the sky plane, so that projection does not hide the density edge".

\subsection{Accretion along the E-W direction}

M07 suggested a possible secondary E-W merger related to lensing mass peaks Nos. 3 and 5. However, these authors doubted the dynamical importance of the structure around the peak No. 5 since this mass peak is poorly significant and the mass-to-light ratio is quite low (but see Okabe \& Umetsu 2008 where the corresponding $\mathrm{C} 3$ peak is quite significant). 


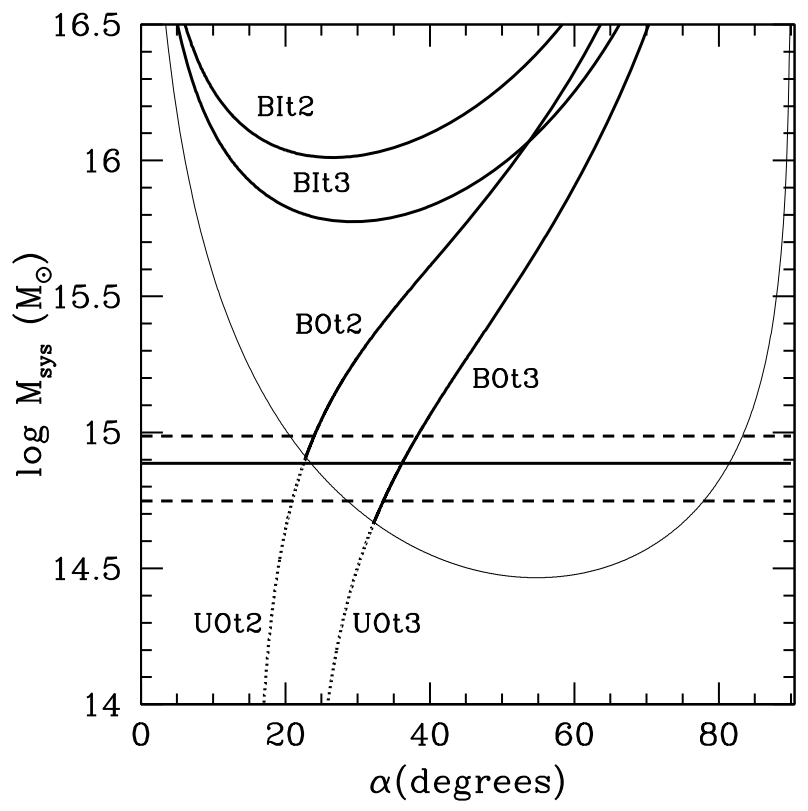

Fig. 15. System mass vs. projection angle for bound outgoing and unbound solutions (solid and dotted curves, respectively) of the two-body model applied to the $\mathcal{N} \mathcal{E} 1+\mathcal{N} \mathcal{E} 2$ and $\mathcal{S} \mathcal{W}$ subsystems. Labels BIt2, BOt2 and UOt2 indicate the curves corresponding to bound incoming, bound outgoing and unbound outgoing solutions assuming that the system is observed at the time $t=0.2$ Gys after the core crossing. Labels BIt3, BOt3 and UOt3 have the same meaning but assuming that the system is observed at $t=0.3$ Gys after the core crossing. The horizontal lines give the observational values of the mass system and its uncertainties. The bound and unbound regions according to the Newtonian criterion are indicated, too (above and below the thin curve, respectively).

We find a strong dynamical evidence of a structure around peak No. 5 (hereafter $\mathcal{E}$ ) at $v \sim 58600 \mathrm{~km} \mathrm{~s}^{-1}$ (i.e. $v_{\mathrm{rf}} \sim$ $-1150 \mathrm{~km} \mathrm{~s}^{-1}$ from the $\mathcal{N} \mathcal{E} 1+\mathcal{N} \mathcal{E} 2$ complex) using the velocity distribution analysis (V1), the velocity dispersion profile (P5) and DS analysis (DS-E). Moreover, $\mathcal{E}$ hosts its luminous galaxy ID 205 close to its center. Comparing $\mathcal{E}$ with $\mathcal{S W}$ we notice that $\mathcal{E}$ lies at a similar velocity distance and at much smaller spatial distance with respect to the $\mathcal{N} \mathcal{E} 1+\mathcal{N} \mathcal{E} 2$ complex. However, since there is no sign of a strong, present interaction from X-ray data, we suspect that $\mathcal{E}$ might be a high-speed remnant of a previous merger.

We also detect a structure (hereafter $\mathcal{W}$ ) located in the western external region not sampled by previous gravitational lensing analyses. This structure is found using both the velocity distribution analysis (part of V2) and the DS analysis (DSW) and is characterized by a velocity $v \sim 59600 \mathrm{~km} \mathrm{~s}^{-1}$ (i.e. $v_{\text {rf }} \sim-300 \mathrm{~km} \mathrm{~s}^{-1}$ from the $\mathcal{N} \mathcal{E} 1+\mathcal{N} \mathcal{E} 2$ complex). It hosts a luminous galaxy close to its center, too (ID 44). Since the $\mathcal{W}$ velocity is similar to that of the $\mathcal{N} \mathcal{E} 1+\mathcal{N} \mathcal{E} 2$ complex and the projected spatial distance is about $2 h_{70}^{-1} \mathrm{Mpc}, \mathcal{W}$ might be a distant subclump well far from the merging, infall phase.

In conclusion we strongly reinforce the possibility of an accretion onto A520 along the E-W direction.

\subsection{Nature of the "massive dark core"}

Finally we discuss the region around lensing mass peak No. 3 for which M07 found a very large mass-to-light ratio claiming for the presence of a massive dark core (but see
Okabe \& Umetsu 2008, where the corresponding C1 peak is not particularly pronounced).

This peak is the only M07 peak for which our analysis of the velocity dispersion profile does not support the presence of an individual structure. The peak of the V4 galaxy distribution is close to peak No. 3, but the V4 group is the only one which does not host any luminous galaxy close to its center. In fact, the two V4 luminous galaxies are located well far in the northern and southern cluster regions (see Fig. 11). Moreover, several galaxies of V4 are likely to be associated with the V4 southern luminous galaxy, i.e. with the $\mathcal{S W}$ structure we discuss in Sect. 5.3. Therefore, only part of galaxies we assign to V4 are likely really connected with the region around peak No. 3 (hereafter we name this minor structure $C$ ).

In conclusion, the existence of an individual, very important structure associated to peak No. 3 is not supported by our kinematical analysis. Rather, we find evidence of two groups centered in that region: the minor $C$ clump (at $v \sim 60900 \mathrm{~km} \mathrm{~s}^{-1}$ ) and the HVG (at $v \sim 62400 \mathrm{~km} \mathrm{~s}^{-1}$ ) suggesting the accretion onto the cluster along the LOS. In agreement with this idea, in the MS+HVG bimodal model of Sect. 5.2 we prefer the bound solution with $\alpha \gtrsim 80$ degrees, with the HVG almost LOS aligned with the MS and infalling onto it. A scenario of a few groups at different velocities agrees with the high velocity dispersion of galaxies we measure around this peak, see also the high velocity ridge in the merging cluster Abell 521 (Ferrari et al. 2003).

Working in this scenario we also look for other possible groups aligned with the LOS. The cluster peak is not well isolated in the velocity space in its high velocity limit, where a few small peaks are present. We analyze the two closest groups of non-member galaxies, hereafter back 1 and back2, formed by 15 and 11 galaxies, respectively (see Fig. 5). Figure 16 shows the spatial distribution of these "background" groups. Galaxies of back1 and back2 have a spatial distribution different from other non-member galaxies (at the $99.96 \%$ c.1. $95 \%$ c.l. according to 2DKS-test). While galaxies of back1 lie at the SW edges of the sampled field and seem to have no connection with the cluster, galaxies of back2 are loosely distributed in central-intermediate cluster regions. Moreover, back 2 group is characterized by a remarkably small velocity dispersion $\sigma_{v} \sim 150 \mathrm{~km} \mathrm{~s}^{-1}$ and a distance of $\Delta z \sim 0.02$ from A520. Thus back 2 might be a very loose group connected with the cluster, since LSS connections are likely found between systems separated by $\Delta z \sim 0.02$ (e.g. Arnaud et al. 2000).

Finally, we discuss the head tail radio galaxy (ID 184). According to Bliton et al (1998) this class of radio galaxies could be related with bulk motion of the intergalactic medium and might indicate the presence of a merger. ID 184 has the tail oriented opposite to the cluster center as expected in a radiogalaxy infalling onto the cluster (see Figs. 1 and 11). Having high velocity $v \sim 60972 \mathrm{~km} \mathrm{~s}^{-1}$, ID 184 is likely not a galaxy of the low velocity structure $\mathcal{E}$, rather it might be connected to the infall along the LOS.

\section{Conclusions: A520 at the crossing of three LSS filaments}

Our findings agree with a scenario where A520 is forming at the crossing of three filaments of the LSS: the NE-SW one, the E$\mathrm{W}$ one, the one along about the LOS. Clusters are expected to form through the accretion along three main filaments according to the LSS formation in the CDM scenario (Shandarin \& Zeldovich 1989, see also beautiful images of simulated clusters 


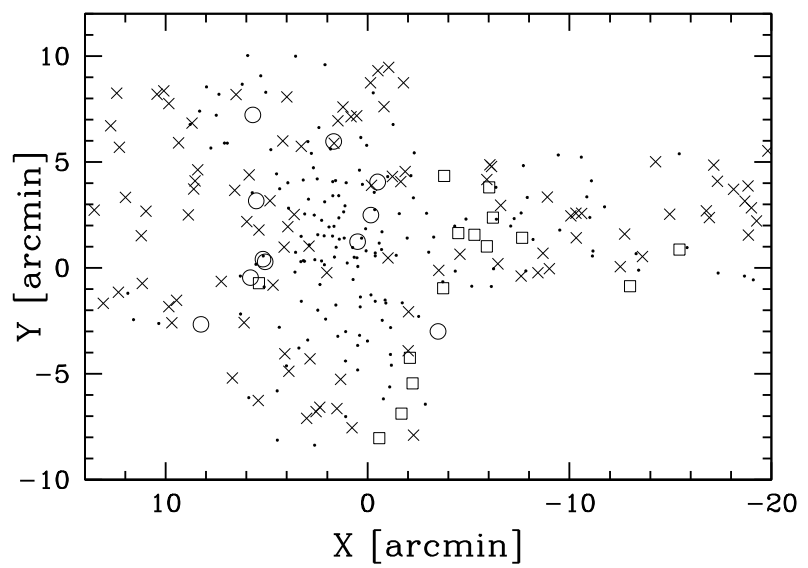

Fig. 16. Spatial distribution on the sky of the 293 galaxies having redshifts in the cluster field. Dots and large symbols indicate member and non member galaxies, respectively. In particular, squares and circles indicate galaxies belonging to back1 and back2 groups.

Springel et al. $2005^{4}$ ). Indeed, a few examples of clusters forming at the crossing of two filaments were already observed (e.g. Arnaud et al. 2000; Cortese et al. 2004; Boschin et al. 2004; Braglia et al. 2007) - see also Matsuda et al. (2005) for a protocluster at the crossing of three filaments.

In this scenario the massive dark core found by M07 analysis would coincide with the peak of the collisional component as shown by X-ray data only due to the particular angle of view of the observer. In fact, the X-ray peak likely traces the potential well of the forming cluster, while the filament aligned with the LOS, projected onto the cluster center, would produce the peak in the 2D mass distribution. Indeed, the hypothesis of a LSS filament projected onto the location of peak No. 3 was already suggested by M07 since is not in obvious contrast with their gravitational lensing data and X-ray data.

Our analysis shows how powerful is the study of the internal cluster dynamics on the basis of velocities and positions of member galaxies. It provides additional information which complements X-ray and gravitational lensing analyses. Other insights into A520 might be recovered from the knowledge of galaxy properties (see e.g. Ferrari et al. 2003; Boschin et al. 2004). In particular, important information comes from the spectral types of member galaxies, since star formation could increase or, alternatively, stop during the merging phase; thus the spectral signatures of past activity are useful to determine the relevant timescales (e.g. Bekki 1999; Terlevich et al. 1999). We are planning further studies of A520 in this perspective.

Acknowledgements. We would like to thank Federica Govoni for the VLA radio image and Maxim Markevitch for the Chandra X-ray image they kindly provided us. We also thank Stefano Borgani for useful discussions.

This publication is based on observations made on the island of La Palma with the Italian Telescopio Nazionale Galileo (TNG), operated by the Fundación Galileo Galilei - INAF (Istituto Nazionale di Astrofisica), and with the Isaac Newton Telescope (INT), operated by the Isaac Newton Group (ING), in the Spanish Observatorio of the Roque de Los Muchachos of the Instituto de Astrofisica de Canarias.

This work was partially supported by a grant from the Istituto Nazionale di Astrofisica (INAF, grant PRIN-INAF2006 CRA ref number 1.06.09.06).

\footnotetext{
4 See

http://www .mpa-garching .mpg.de/galform/millennium/
}

\section{References}

Abell, G. O., Corwin, H. G. Jr., \& Olowin, R. P. 1989, ApJS, 70, 1 Arnaud, M., Maurogordato, S., Slezak, E., \& Rho, J. 2000, A\&A, 355, 461

Ashman, K. M., Bird, C. M., \& Zepf, S. E. 1994, AJ, 108, 2348

Bardelli, S., Zucca, E., Vettolani, G., et al. 1994, MNRAS, 267, 665

Barrena, R., Boschin, W., Girardi, M., \& Spolaor, M. 2007a, A\&A, 467, 37

Barrena, R., Boschin, W., Girardi, M., \& Spolaor, M. 2007b, A\&A, 469, 861

Beers, T. C., Geller, M. J., \& Huchra, J. P. 1982, ApJ, 257, 23

Beers, T. C., Flynn, K., \& Gebhardt, K. 1990, AJ, 100, 32

Beers, T. C., Forman, W., Huchra, J. P., Jones, C., \& Gebhardt, K. 1991, AJ, 102, 1581

Beers, T. C., Gebhardt, K., Huchra, J. P., et al. 1992, ApJ, 400, 410

Bekki, K. 1999, ApJ, 510, L15

Bertin, E., \& Arnouts, S. 1996, A\&AS, 117, 393

Bird, C. M. 1994, ApJ, 422, 480

Bird, C. M., \& Beers, T. C. 1993 , AJ, 105, 1596

Biviano, A., \& Katgert, P. 2004, A\&A, 424, 779

Biviano, A., Katgert, P., Thomas, T., \& Adami, C. 2002, A\&A, 387, 8

Bliton, M., Rizza, E., Burns, J. O., Owen, F. N., \& Ledlow, M. J. 1998, MNRAS, 301,609

Böhringer, H., \& Schuecker, P. 2002, in Merging Processes in Galaxy Clusters, ed. L. Feretti, I. M. Gioia, \& G. Giovannini, ASSL, 272, 133

Borgani, S., Girardi, M., Carlberg, R. G., Yee, H. K. C., \& Ellingson, E. 1999, ApJ, 527, 561

Boschin, W., Girardi, M., Barrena, R., et al. 2004, A\&A, 416, 839

Boschin, W., Girardi, M., Spolaor, M., \& Barrena, R. 2006, A\&A, 449, 461

Boschin, W., Barrena, R., Girardi, M., \& Spolaor, M. 2008, A\&A, 487, 33

Braglia, F., Pierini, D., \& Böhringer, H. 2007, A\&A, 470, 425

Bullock, J. S., Kolatt, T. S., Sigad, Y., et al. 2001, MNRAS, 321, 559

Buote, D. A. 2002, in Merging Processes in Galaxy Clusters, eds. L. Feretti, I. M. Gioia, \& G. Giovannini, ASSL, 272, 79

Burstein, D., \& Heiles, C. 1982, AJ, 87, 1165

Carlberg, R. G., Yee, H. K. C., Ellingson, E., et al. 1996, ApJ, 462, 32

Carlberg, R. G., Yee, H. K. C., \& Ellingson, E. 1997, ApJ, 478, 462

Cooray, A. R., Grego, L., Holzapfel, W. L., Joy, M., \& Carlstrom, J. E. 1998, AJ, 115,1388

Cortese, L., Gavazzi, G., Boselli, A., Iglesias-Paramo, J., \& Carrasco, L. 2004, A\&A, 425, 429

Dahle, H., Kaiser, N., Irgens, R. J., Lilje, P. B., \& Maddox, S. J. 2002, ApJS, 139,313

Danese, L., De Zotti, C., \& di Tullio, G. 1980, A\&A, 82, 322

Dolag, K., Bartelmann, M., Perrotta, F., et al. 2004, A\&A, 416, 853

Dressler, A., \& Shectman, S. A. 1988, AJ, 95, 985

Ebeling, H., Voges, W., Böhringer, H., et al. 1996, MNRAS, 281, 799

Ellingson, E., \& Yee, H. K. C. 1994, ApJS, 92,33

Fadda, D., Girardi, M., Giuricin, G., Mardirossian, F., \& Mezzetti, M. 1996, ApJ, 473,670

Fasano, G., \& Franceschini, A. 1987, MNRAS, 225, 155

Feretti, L. 1999, MPE Report No. 271

Feretti, L. 2002, The Universe at Low Radio Frequencies, Proc. IAU Symp. 199, held 30 Nov.-4 Dec. 1999, Pune, India, ed. A. Pramesh Rao, G. Swarup, \& Gopal-Krishna, 133

Feretti, L. 2005, X-ray and Radio Connections, ed. L. O. Sjouwerman, \& K. K. Dyer, held 3-6 February 2004 in Santa Fe, New Mexico, USA. Published electronically by NRAO, http://www. aoc.nrao. edu/events/xraydio

Feretti, L., Gioia I. M., \& Giovannini G. (eds.) 2002, Merging Processes in Galaxy Clusters (Dordrecht: Kluwer Academic Publisher), Astrophysics and Space Science Library, 272

Ferrari, C., Maurogordato, S., Cappi, A., \& Benoist C. 2003, A\&A, 399, 813

Flores, R. A., Quintana, H., \& Way, M. J. 2000, ApJ, 532, 206

Gioia, I. M., \& Luppino, G. A. 1994, ApJS, 94, 583

Gioia, I. M., Henry, J. P., Maccacaro, T., et al. 1990, ApJ, 356, 35

Giovannini, G., \& Feretti, L. 2002, in Merging Processes in Galaxy Clusters, ed.

L. Feretti, I. M. Gioia, \& G. Giovannini, ASSL, 272, 197

Giovannini, G., Tordi, M., \& Feretti, L. 1999, New Astronomy, 4, 141

Girardi, M., \& Biviano, A. 2002, in Merging Processes in Galaxy Clusters, ed.

L. Feretti, I. M. Gioia, \& G. Giovannini, ASSL, 272, 39

Girardi, M., \& Mezzetti, M. 2001, ApJ, 548, 79

Girardi, M., Biviano, A., Giuricin, G., Mardirossian, F., \& Mezzetti, M. 1993, ApJ, 404, 38

Girardi, M., Fadda, D., Giuricin, G., et al. 1996, ApJ, 457, 61

Girardi, M., Giuricin, G., Mardirossian, F., Mezzetti, M., \& Boschin, W. 1998, ApJ, 505, 74

Girardi, M., Demarco, R., Rosati, P., \& Borgani, S. 2005, A\&A, 442, 29

Girardi, M., Boschin, W., \& Barrena, R. 2006, A\&A, 455, 45

Govoni, F., Ensslin, T. A., Feretti, L., \& Giovannini, G. 2001a, A\&A, 369, 441

Govoni, F., Feretti, L., Giovannini, G., et al. 2001b, A\&A, 376, 803 
Govoni, F., Markevitch, M., Vikhlinin, A., et al. 2004, ApJ, 605, 695

Gregory, S. A., \& Thompson, L. A. 1984, ApJ, 286, 422

Gullixson, C. A. 1992, in Astronomical CCD Observing and Reduction techniques, ed. S. B. Howell, ASP Conf. Ser., 23, 130

Kennicutt, R. C. 1992, ApJS, 79, 225

Le Fèvre, O., Hammer, F., Angonin, M. C., Gioia, I. M., \& Luppino, G. A. 1994 ApJ, 422, 5

Limber, D. N., \& Mathews, W. G. 1960, ApJ, 132, 286

Lewis, A. D., Ellingson, E., Morris, S. L., \& Carlberg, R. G. 1999, ApJ, 517, 587

Lubin, L. M., Postman, M., \& Oke, J. B. 1998, AJ, 116, 643

Mahdavi, A., Hoekstra, H., Babul, A., Balam, D. D., \& Capak, P. L. 2007, ApJ, 668, 806 [M07]

Malumuth, E. M., Kriss, G. A., Dixon, W. Van Dyke, Ferguson, H. C., \& Ritchie, C. 1992, AJ, 104, 495

Markevitch, M., Gonzalez, A. H., David, L., et al. 2002, ApJ, 567, 27

Markevitch, M., Govoni, F., Brunetti, G., \& Jerius, D. 2005, ApJ, 627, 733

Matsuda, Y., Yamada, T., Hayshino, T., et al. 2005, ApJ, 634, 125

Navarro, J. F., Frenk, C. S., \& White, S. D. M. 1997, ApJ, 490, 493

Newberry, M. V., Kirshner, R. P., \& Boroson, T. A. 1988, ApJ, 335, 629

Okabe, N., \& Umetsu, K. 2008, PASJ, 60, 345

Pisani, A. 1993, MNRAS, 265, 706
Pisani, A. 1996, MNRAS, 278, 697

Press, W. H., Teukolsky, S. A., Vetterling, W. T., \& Flannery, B. P. 1992, in

Numerical Recipes (Cambridge University Press), 2nd edn

Proust, D., Cuevas, H., Capelato, H. V., et al. 2000, A\&A, 335, 443

Quintana, H., Carrasco, E. R., \& Reisenegger, A. 2000, AJ, 120, 511

Roettiger, K., Loken, C., \& Burns, J. O. 1997, ApJS, 109, 307

Sarazin, C. L. 2002, in Merging Processes in Galaxy Clusters, ed. L. Feretti, I. M. Gioia, \& G. Giovannini, ASSL, 272, 1

Schuecker, P., Böhringer, H., Reiprich, T. H., \& Feretti, L. 2001, A\&A, 378, 408

Shandarin, S. F., \& Zeldovich Ya. B. 1989, Rev. Mod. Phys., 61, 185

Shapiro, S. S., \& Wilk, M. B. 1965, Biometrika, 52, 591

Springel, V, White, S. D., Jenkins, A., et al., Nature, 435, 629

Terlevich, A. I., Kuntschner, H., Bower, R. G., Caldwell, N., \& Sharples, R. M. 1999, MNRAS, 310, 445

The, L. S., \& White, S. D. M. 1986, AJ, 92, 1248

Thompson, L. A. 1982, in IAU Symp. 104, Early Evolution of the Universe and the Present Structure, ed. G. O. Abell \& G. Chincarini (Dordrecht: Reidel) Tonry, J., \& Davis, M. 1979, ApJ, 84, 1511

Yee, H. K. C., Ellingson, E., \& Carlberg, R. G. 1996, ApJS, 102, 269

Wainer, H., \& Schacht, S. 1978, Psychometrika, 43, 203

Wright, E. L. 2006, PASP, 118, 1711 